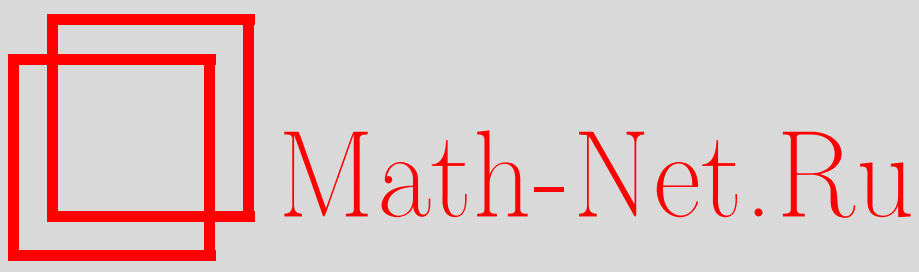

В. Е. Федоров, Голоморфные разрешающие полугруппы уравнений соболевского типа в локально выпуклых пространствах, Матем. сб., 2004, том 195, номер 8, $131-$ 160

DOI: https://doi.org/10.4213/sm841

Использование Общероссийского математического портала Math-Net.Ru подразумевает, что вы прочитали и согласны с пользовательским соглашением

http://www.mathnet.ru/rus/agreement

Параметры загрузки:

IP: 54.198 .55 .26

26 апреля 2023 г., 16:33:49 


\section{Голоморфные разрешающие полугруппы уравнений соболевского типа в локально выпуклых пространствах}

Исследуются вопросы существования сильно голоморфных в секторе экспоненциально ограниченных полугрупп линейного уравнения соболевского типа

$$
L \dot{u}=M u
$$

с непрерьвньм оператором $L: \mathfrak{U} \rightarrow \mathfrak{F}, \operatorname{ker} L \neq\{0\}$, и замкнутьп и плотно определенным оператором $M: \operatorname{dom} M \rightarrow \mathfrak{F}, \mathfrak{U}, \mathfrak{F}$ - секвенциально полные локально вьпуклые пространства. Показано, что для существования таких полугрупп, вырождающихся на $M$-присоединенных векторах оператора $L$ высоты не больше $p$, и существования пар инвариантных подпространств операторов $L$ и $M$ необходимьм и достаточным является условие сильной $(L, p)$-секториальности оператора $M$, обобщающее известное условие секториальности. Получено обобщение теоремы Иосиды, а также теорем о существовании голоморфных полугрупп уравнения (1) в банаховых пространствах. Полученные результаты применяются при исследовании ослабленной задачи Коши для уравнения (1) и для соответствующего неоднородного уравнения. Приложением абстрактных результатов является теорема о достаточных условиях разрешимости задачи Коши для одного класса уравнений в пространствах Фреше специального вида. Этот результат используется при исследовании периодической задачи Коши для уравнения в частных производных со смещением по пространственной переменной, не разрешенного относительно производной по времени.

Библиограф̆ия: 18 названий.

\section{Введение}

Пусть $\mathfrak{U}, \mathfrak{F}, \mathfrak{V}$ - отделимые секвенциально полные локально вьпуклые линейные топологические пространства. Обозначим через $\mathscr{L}(\mathfrak{U} ; \mathfrak{F})$ векторное пространство линейных непрерьвных операторов из $\mathfrak{U}$ в $\mathfrak{F}$, а через $\mathrm{Cl}(\mathfrak{U} ; \mathfrak{F})$ - множество линейных замкнутых операторов с плотными в $\mathfrak{U}$ областями определения, действующих в пространство $\mathfrak{F}$. В случае $\mathfrak{U}=\mathfrak{F}$ будем использовать обозначения $\mathscr{L}(\mathfrak{U})=\mathscr{L}(\mathfrak{U} ; \mathfrak{F}), \mathrm{Cl}(\mathfrak{U})=\mathrm{Cl}(\mathfrak{U} ; \mathfrak{F})$.

Рассмотрим уравнение на пространстве $\mathfrak{V}$

$$
\dot{v}(t)=A v(t)
$$

Сушествует множество эквивалентных формулировок теоремы о существовании голоморфной полугруппы уравнения (0.1) (см. [1], [2; гл. 1, §3], [3; гл. 5, §6], [4]),

Работа поддержана грантом Минобразования России (№ PD02-1.1-82) на проведение молодьми ученьми научных исследований и грантом для молодых ученых Правительства Челябинской области. 
которую в дальнейшем мы будем называть теоремой Иосиды [1; гл. IX, § 10]. Наиболее удобной представляется следующая формулировка: голоморфная в секторе полугруппа уравнения (0.1) сушествует тогда и только тогда, когда оператор $A$ секториален. Секториальнылм называется (см. [2]-[4]) оператор $A \in \mathrm{Cl}(\mathfrak{V})$, для которого семейство непрерывных операторов

$$
\left\{(\mu-a)(\mu I-A)^{-1}:|\arg (\mu-a)|<\theta\right\}
$$

эквинепрерывно при некоторых $a \in \mathbb{R}, \theta \in(\pi / 2, \pi)$.

Следуя [5; гл. I, §3], ослабленной задачей Коши для уравнения (0.1) будем называть задачу отыскания решения уравнения $v$ из класса функций $C^{1}\left(\mathbb{R}_{+} ; \mathfrak{V}\right) \cap$ $C\left(\overline{\mathbb{R}}_{+} ; \mathfrak{V}\right), \overline{\mathbb{R}}_{+}=\{0\} \cup \mathbb{R}_{+}$, удовлетворяющего условию $v(0)=v_{0} . \mathrm{B}[5]$ показано, что достаточным условием разрешимости ослабленной задачи Коши для уравнения (0.1) при любом $v_{0} \in \mathfrak{V}$ является секториальность оператора $A$.

В случае непрерывной обратимости оператора $L \in \mathscr{L}(\mathfrak{U} ; \mathfrak{F})$ уравнению $(0.1)$ эквивалентно уравнение

$$
L \dot{u}(t)=M u(t)
$$

с оператором $M \in \mathrm{Cl}(\mathfrak{U} ; \mathfrak{F})$. Однако это не так, если $\operatorname{ker} L \neq\{0\}$, при этом уравнение (0.2), следуя устоявшейся традиции, будем назьвать уравнением соболевского типа. О неуменьшаюшемся интересе к таким уравнениям говорит количество вьшедших в последнее время посвяшенных им монографий [6]-[8].

Необходимо отметить, что полугруппа, разрешаюшая уравнение соболевского типа, вырождается на некотором подпространстве. Это подпространство, ядро единицы полугруппы, в дальнейшем будем называть ядром nолугрупnьь. Ранее голоморфные полугруппы уравнения (0.2) исследовались в [7; гл. III], однако лишш в случае банаховых пространств и совпадения ядра полугруппы с ядром оператора L. В работах [9], [10] были изучены голоморфные полугрупшы также в банаховых пространствах при условии $(L, p)$-секториальности оператора $M$, которое допускает вырождение полугруппы не только на ядре оператора $L$, но и на его $M$-присоединенных векторах высоты не больше $p$.

Настоящая работа продолжает начатое в [9], [10] исследование голоморфных полугрупп вырожденного $(\operatorname{ker} L \neq\{0\})$ уравнения (0.2). Перечислим основные новые моменты, послу жившие стимулом для ее написания.

1) Следуя К. Иосиде, удалось показать, что и для вырожденного уравнения (0.2) результаты об экспоненциально ограниченных голоморфных разрешающих полугруппах переносятся со случая банаховых на случай локально выпуклых пространств по сути без изменений. Понятие сильно $(L, p)$-секториального оператора при этом обобщено на случай локально вьпуклых пространств путем замены условий на рост нормы правой и левой $(L, p)$-резольвент в секторе на условия эквинепрерьвности соответствующих семейств операторов.

2 ) Получены оценки на рост не только полугрупшы уравнения (0.2), но и ее производных. Даже для случая банаховых пространств это новый результат.

$3)$ В работе показано, что при условии $(L, p)$-секториальности оператора $M$ образ разрешаюшей полугруппы уравнения (0.2) совпадает с его фазовым пространством, т.е. множеством допустимых начальных значений ослабленной задачи Коши для уравнения. Ранее даже в случае банаховых пространств такой результат 
был доказан лишь при дополнительном условии бесконечной дифференцируемости всех решений (см. [10; док-во теоремы 3.1$])$.

4) Как и в случае банаховых пространств [10], доказана необходимость и достаточность условия сильной $(L, p)$-секториальности оператора $M$ для существования разрешаюших голоморфных полугрупп на пространствах $\mathfrak{U}$ и $\mathfrak{F}$ и инвариантных пар подпространств операторов $L$ и $M$. Этот результат обобшает теорему Иосиды (см. §7). Кроме того, показана особая роль случая полурефлексивных пространств, в котором упомянутое обобщение можно получить, используя более слабое условие $(L, p)$-секториальности оператора $M$.

$5)$ На основе результатов о полугруппах уравнения (0.2) получены условия разрешимости ослабленной задачи Коши для неоднородного вырожденнного уравнения $L \dot{u}(t)=M u(t)+f(t)$.

6) Абстрактные результаты используются при исследовании рассматриваемого в специальном пространстве Фреше уравнения $P(A) \dot{u}(t)=Q(A) u(t)+f(t)$, где $A-$ самосопряженный замкнутый плотно определенный в некотором банаховом пространстве оператор, $P, Q$ - целые функции. К последнему уравнению, в свою очередь, сведена периодическая по пространственной переменной задача Коши для уравнения в частных производных со смешением по пространственной переменной, не разрешенного относительно производной по времени.

Обратим внимание, что в случае непрерьвной обратимости оператора $L$ уравнение (0.2) можно редуцировать к уравнению $\dot{u}(t)=L^{-1} M u(t)$ на пространстве $\mathfrak{U}$ или к уравнению $\dot{f}(t)=M L^{-1} f(t), f(t)=L u(t)$, на пространстве $\mathfrak{F}$. Тем самьм в случае секториальности оператора $L^{-1} M$ (или, что равносильно, оператора $M L^{-1}$ ) паре операторов соответствует пара разрешающих полугрупп: одна задана на пространстве $\mathfrak{U}$, другая - на пространстве $\mathfrak{F}$. Чтобы сохранить такую симметрию пространств и в вырожденном случае, наряду с уравнением $(0.2)$ на пространстве $\mathfrak{U}$ будем рассматривать эквивалентное ему уравнение на пространстве $\mathfrak{F}$

$$
L_{\alpha}^{L}(M) \dot{f}(t)=M(\alpha L-M)^{-1} f(t), \quad \alpha \in \rho^{L}(M) .
$$

В $\S 7$ именно наличие двух полугрупп на различных пространствах позволяет получить полное обобщение теоремы Иосиды.

\section{§1. Относительно $p$-секториальный оператор}

Напомним, что локально выпуклые пространства $\mathfrak{U}$ и $\mathfrak{F}$ секвенциально полны, операторы $L \in \mathscr{L}(\mathfrak{U} ; \mathfrak{F}), M \in \mathrm{Cl}(\mathfrak{U} ; \mathfrak{F})$. Кроме того, заметим, что когда доказательства утверждений, касающихся пространства $\mathfrak{F}$, не отличаются от доказательств аналогичных утверждений, касающихся пространства $\mathfrak{U}$, мы будем их опускать, не оговаривая это особо.

Введем следуюшие обозначения:

$$
\begin{gathered}
\rho^{L}(M)=\left\{\mu \in \mathbb{C}:(\mu L-M)^{-1} \in \mathscr{L}(\mathfrak{F} ; \mathfrak{U})\right\}, \\
R_{\mu}^{L}(M)=(\mu L-M)^{-1} L, \quad L_{\mu}^{L}(M)=L(\mu L-M)^{-1}, \\
R_{(\lambda, p)}^{L}(M)=\prod_{k=0}^{p} R_{\lambda_{k}}^{L}(M), \quad L_{(\lambda, p)}^{L}(M)=\prod_{k=0}^{p} L_{\lambda_{k}}^{L}(M) .
\end{gathered}
$$


В дальнейших рассмотрениях нам понадобятся тождества

$$
\begin{gathered}
(\mu-\lambda)(\lambda L-M)^{-1} L(\mu L-M)^{-1}=(\lambda L-M)^{-1}-(\mu L-M)^{-1}, \\
L(\mu L-M)^{-1} M u=M(\mu L-M)^{-1} L u, \quad u \in \operatorname{dom} M .
\end{gathered}
$$

Пусть $\operatorname{ker} L \neq\{0\}$, тогда упорядоченное множество векторов $\left\{\varphi_{0}, \varphi_{1}, \ldots\right\}$ называется цепочкой $M$-присоединенных векторов вектора $\varphi_{0} \in \operatorname{ker} L \backslash\{0\}$, если

$$
L \varphi_{k+1}=M \varphi_{k}, \quad k=0,1, \ldots, \quad \varphi_{l} \notin \operatorname{ker} L, \quad l=1,2, \ldots .
$$

Порядковый номер $k$ вектора в цепочке (он совпадает с нижним индексом $k$, где $k=0,1,2, \ldots)$ будем называть его въьсотой.

ЗАмечание 1.1. Цепочка $M$-присоединенных векторов оператора $L$, соответствуюшая вектору $\varphi_{0} \in \operatorname{ker} L \backslash\{0\},-$ это в точности цепочка присоединенных векторов к собственному вектору $\varphi_{0}$ пучка операторов $L-\lambda M$ в точке $\lambda=0$ $[11 ;$ гл. V, §9].

Доказательства следуюших двух лемм можно найти в [12].

Лемма 1.1. Пусть $\varphi_{l}-M$-присоединенный вектор оператора $L$ высоты $l \geqslant 1$. Тогда для всех $\mu \in \rho^{L}(M)$ имеем $-R_{\mu}^{L}(M) \varphi_{l}=\varphi_{l-1}+\mu \varphi_{l-2}+\cdots+$ $\mu^{l-1} \varphi_{0}$.

Лемма 1.2. Если $\lambda=\left(\lambda_{0}, \ldots, \lambda_{p}\right), \mu=\left(\mu_{0}, \ldots, \mu_{p}\right) \in\left(\rho^{L}(M)\right)^{p+1}, m o$

i) $\operatorname{ker} R_{(\lambda, p)}^{L}(M)$ есть линейная оболочка множества $M$-присоединенных виссоты не большей $р$ векторов оператора $L, \operatorname{im} R_{(\lambda, p)}^{L}(M)=$ $\operatorname{im} R_{(\mu, p)}^{L}(M)$

ii) $\operatorname{ker} L_{(\lambda, p)}^{L}(M)=\left\{M \varphi: \varphi \in \operatorname{ker} R_{(\lambda, p)}^{L}(M) \cap \operatorname{dom} M\right\}, \operatorname{im} L_{(\lambda, p)}^{L}(M)=$ $\operatorname{im} L_{(\mu, p)}^{L}(M)$.

Обозначим через $\mathfrak{U}^{0}\left(\mathfrak{F}^{0}\right)$ ядро $\operatorname{ker} R_{(\mu, p)}^{L}(M)\left(\operatorname{ker} L_{(\mu, p)}^{L}(M)\right)$, а через $L_{0}\left(M_{0}\right)$ сужение оператора $L(M)$ на $\mathfrak{U}^{0}\left(\operatorname{dom} M_{0}=\mathfrak{U}^{0} \cap \operatorname{dom} M\right)$ соответственно. Очевидно, что $L_{0} \in \mathscr{L}\left(\mathfrak{U}^{0} ; \mathfrak{F}^{0}\right), M_{0}: \operatorname{dom} M_{0} \rightarrow \mathfrak{F}^{0}$.

Будем говорить, что семейство операторов $\{\Phi(x) \in \mathscr{L}(\mathfrak{U} ; \mathfrak{F}): x \in X\}, X-$ множество индексов, әквинепрерывно, если для любой непрерывной на $\mathfrak{F}$ полунормы $r(\cdot)$ сушествует непрерывная на $\mathfrak{U}$ полунорма $q(\cdot)$ такая, что $\forall x \in X, u \in \mathfrak{U}$ $r(\Phi(x) u) \leqslant q(u)$.

ОПРЕДЕЛЕНИЕ 1.1. Оператор $M$ назьвается $(L, p)$-секториальныц. если выполняются условия:

i) при некоторых $a \in \mathbb{R}, \theta \in(\pi / 2, \pi) S_{a, \theta}^{L}(M)=\{\mu \in \mathbb{C}:|\arg (\mu-a)|<\theta\} \subset$ $\rho^{L}(M)$

ii) эквинепрерывны семейства операторов

$$
\begin{aligned}
& \left\{R_{(\mu, p)}^{L}(M) \prod_{k=0}^{p}\left(\mu_{k}-a\right): \mu=\left(\mu_{0}, \ldots, \mu_{p}\right) \in\left(S_{a, \theta}^{L}(M)\right)^{p+1}\right\} \\
& \left\{L_{(\mu, p)}^{L}(M) \prod_{k=0}^{p}\left(\mu_{k}-a\right): \mu=\left(\mu_{0}, \ldots, \mu_{p}\right) \in\left(S_{a, \theta}^{L}(M)\right)^{p+1}\right\} .
\end{aligned}
$$


ЗАмЕчАниЕ 1.2. Пусть существует оператор $L^{-1} \in \mathscr{L}(\mathfrak{F} ; \mathfrak{U})$. Если оператор $L^{-1} M$ (или оператор $M L^{-1}$ ) секториален, то оператор $M(L, p)$-секториален.

Следуюшие две леммы доказываются так же, как и аналогичные результаты в случае банаховых пространств [10].

Лемма 1.3. Пусть оператор $M(L, p)$-секториален. Тогда

i) множество $\operatorname{ker} R_{(\mu, p)}^{L}(M)$ состоит в точности из $M$-присоединенных векторов оператора $L$ висоты не больше $p$;

ii) $\operatorname{ker} R_{(\mu, p)}^{L}(M) \cap \operatorname{im} R_{(\mu, p)}^{L}(M)=\{0\}, \operatorname{ker} L_{(\mu, p)}^{L}(M) \cap \operatorname{im} L_{(\mu, p)}^{L}(M)=\{0\}$.

Через $\mathfrak{U}^{1}\left(\mathfrak{F}^{1}\right)$ обозначим замыкание $\operatorname{im} R_{(\mu, p)}^{L}(M)\left(\operatorname{im} L_{(\mu, p)}^{L}(M)\right)$, через $\widetilde{\mathfrak{U}}(\widetilde{\mathfrak{F}})$ замыкание $\mathfrak{U}^{0}+\operatorname{im} R_{(\mu, p)}^{L}(M)\left(\mathfrak{F}^{0}+\operatorname{im} L_{(\mu, p)}^{L}(M)\right)$ в топологии пространства $\mathfrak{U}(\mathfrak{F})$.

Лемма 1.4. Пусть оператор $M(L, p)$-секториален. Тогда

i) при любъхх $u \in \mathfrak{U}^{1}, f \in \mathfrak{F}^{1}$

$$
\lim _{\mu \rightarrow+\infty}\left(\mu R_{\mu}^{L}(M)\right)^{p+1} u=u, \quad \lim _{\mu \rightarrow+\infty}\left(\mu L_{\mu}^{L}(M)\right)^{p+1} f=f
$$

ii) $\widetilde{\mathfrak{U}}=\mathfrak{U}^{0} \oplus \mathfrak{U}^{1}, \widetilde{\mathfrak{F}}=\mathfrak{F}^{0} \oplus \mathfrak{F}^{1}$.

Проектор вдоль $\mathfrak{U}^{0}\left(\mathfrak{F}^{0}\right)$ на $\mathfrak{U}^{1}\left(\mathfrak{F}^{1}\right)$ имеет следующий вид:

$$
\widetilde{P}=\underset{\mu \rightarrow+\infty}{\mathrm{s}-\lim _{i \rightarrow \infty}}\left(\mu R_{\mu}^{L}(M)\right)^{p+1} \quad\left(\widetilde{Q}=\underset{\mu \rightarrow+\infty}{\mathrm{s}-\lim _{\mu \rightarrow+}}\left(\mu L_{\mu}^{L}(M)\right)^{p+1}\right) .
$$

В дальнейшем нам понадобится степенное ограничение на рост оператор-функции $(\mu L-M)^{-1}$ на бесконечности, которое следует из $(L, p)$-секториальности оператора $M$. В случае банаховых пространств этот факт доказан в [10; лемма 1.3], хотя формулировка леммы 1.3 приведена в [10] не совсем точно. Уточним ее сразу для локально выпуклых пространств.

Положим $B_{r}(z)=\{\mu \in \mathbb{C}:|\mu-z| \leqslant r\}$.

Лемма 1.5. Пусть оператор $M(L, p)$-секториален. Тогда при любом $\varepsilon>0$ семейство операторов $\left\{\mu^{-p}(\mu L-M)^{-1}: \mu \in S_{a, \theta}^{L}(M) \backslash B_{|a|+\varepsilon}(0)\right\}$ әквинепрерьвноо.

Мы докажем чуть более слабую оценку, но на более шшроком множестве.

Лемма 1.6. Пусть оператор $M(L, p)$-секториален. Тогда при любых $\delta_{1}, \delta_{2}>0$ семейство операторов

$$
\left\{\mu^{-p-1}(\mu L-M)^{-1}: \mu \in S_{a, \theta}^{L}(M) \backslash\left(B_{\delta_{1}}(a) \cup B_{\delta_{2}}(0)\right)\right\}
$$

эквинепрерьвно. 
ДокаЗАТЕЛЬСТво. Возьмем $\beta \in S_{a, \theta}^{L}(M)$. Тождество

$$
\begin{aligned}
(\mu L-M)^{-1}= & \sum_{k=0}^{p}(\beta-\mu)^{k}\left(R_{\beta}^{L}(M)\right)^{k}(\beta L-M)^{-1} \\
& +(\beta-\mu)^{p+1} R_{\mu}^{L}(M)\left(R_{\beta}^{L}(M)\right)^{p}(\beta L-M)^{-1}
\end{aligned}
$$

можно доказать последовательным применением тождества (1.1). Из определения 1.1 следует, что для всякой непрерывной в $\mathfrak{U}$ полунормы $q(\cdot)$ существуют непрерывные в $\mathfrak{F}$ полунормы $r_{k}(\cdot), k=\overline{0, p+1}$, такие, что для всех $f \in \mathfrak{F}$ и $\mu \in S_{a, \theta}^{L}(M) \backslash\left(B_{\delta_{1}}(a) \cup B_{\delta_{2}}(0)\right)$

$$
\begin{aligned}
& q\left(\mu^{-p-1}(\mu L-M)^{-1} f\right) \leqslant|\mu|^{-p-1} \sum_{k=0}^{p}(|\beta|+|\mu|)^{k} r_{k}(f) \\
& \quad+\frac{(|\beta|+|\mu|)^{p+1}}{|\mu|^{p+1}|\mu-a|}|\beta-a|^{-p} r_{p+1}(f) \\
& \leqslant|\mu|^{-p-1} \sum_{k=0}^{p}(|\beta|+|\mu|)^{k} r_{k}(f)+\frac{\left(1+|\beta| / \delta_{2}\right)^{p+1}}{\delta_{1}}|\beta-a|^{-p} r_{p+1}(f) \\
& \leqslant \sum_{k=0}^{p+1} c_{k} r_{k}(f)=r(f),
\end{aligned}
$$

где $r(\cdot)$ - непрерывная на пространстве $\mathfrak{F}$ полунорма.

\section{§ 2. Существование голоморфных полугрупп}

ОПРЕДЕЛЕНИЕ 2.1. Отображение $U(\cdot): \Sigma \rightarrow \mathscr{L}(\mathfrak{U}), \Sigma=\left\{\tau \in \mathbb{C}: \psi_{1}<\right.$ $\left.\arg \tau<\psi_{2}\right\},-\pi<\psi_{1}<\psi_{2}<\pi$, называется сильно голоморфной в секторе $\Sigma$ разрешающей полугруппой уравнения (0.2), если

i) $U(\sigma) U(\tau)=U(\sigma+\tau) \forall \sigma, \tau \in \Sigma$;

ii) для любого $u_{0} \in \mathfrak{U}$ функция $u(\tau)=U(\tau) u_{0}$ дифференцируема во всех точках $\tau \in \Sigma$ в топологии пространства $\mathfrak{U}$ и удовлетворяет уравнению $(0.2)$.

Наличие единицы у полугрупшы не постулируется.

Сильно голоморфную в секторе $\Sigma$ полугруппу назовем әкспоненциально ограниченной, если при некотором $a \in \mathbb{R}$ семейство операторов $\left\{e^{-a \tau} U(\tau): \tau \in \Sigma\right\}$ эквинепрерывно.

Tеорема 2.1. Пусть оператор $M$ (L, p)-секториален. Тогда существует сильно голоморфная в секторе $\Sigma_{\theta}=\{\tau \in \mathbb{C}:|\arg \tau|<\theta-\pi / 2\}$ разрешающая полугруппа $\left\{U(\tau) \in \mathscr{L}(\mathfrak{U}): \tau \in \Sigma_{\theta}\right\} \quad\left(\left\{F(\tau) \in \mathscr{L}(\mathfrak{F}): \tau \in \Sigma_{\theta}\right\}\right)$ уравнения $(0.2)((0.3))$. Кроме того, при любом $\delta \in(0, \theta-\pi / 2)$ семейство операторов $\left\{U(\tau): \tau \in \Sigma_{\theta-\delta}\right\} \quad\left(\left\{F(\tau): \tau \in \Sigma_{\theta-\delta}\right\}\right)$ экспоненциально ограничено, и при любом $R>0$ әкспоненииально ограничень семейства операторов $\left\{\tau^{n} U^{(n)}(\tau): \tau \in \Sigma_{\theta-\delta} \cap B_{R}(0)\right\} \quad\left(\left\{\tau^{n} F^{(n)}(\tau): \tau \in \Sigma_{\theta-\delta} \cap B_{R}(0)\right\}\right), n \in \mathbb{N}$. 
ДокАЗАТЕЛЬСтво. Операторы полугрупп имеют вид

$$
U(\tau)=\frac{1}{2 \pi i} \int_{\Gamma} R_{\mu}^{L}(M) e^{\mu \tau} d \mu, \quad F(\tau)=\frac{1}{2 \pi i} \int_{\Gamma} L_{\mu}^{L}(M) e^{\mu \tau} d \mu .
$$

Контур Г при этом взят следующий:

$$
\Gamma=\{\mu \in \mathbb{C}: \mu=a+\varepsilon-|x|+i x \operatorname{tg} \theta, x \in \mathbb{R}\} .
$$

Здесь $\varepsilon>0, \varepsilon \neq-a$, где $a-$ из определения 1.1. Тогда при некоторых $\delta_{1}, \delta_{2}>0$ $\Gamma \subset S_{a, \theta}^{L}(M) \backslash\left(B_{\delta_{1}}(a) \cup B_{\delta_{2}}(0)\right)$. Используя лемму 1.6 , нетрудно показать сходимость интегралов

$$
G_{k}^{\tau} u=\frac{1}{2 \pi i} \int_{\Gamma} \mu^{k} R_{\mu}^{L}(M) e^{\mu \tau} u d \mu, \quad k \in\{0\} \cup \mathbb{N},
$$

равномерную по параметру $\tau \in B_{R}(0) \cap \Sigma_{\theta-\delta} \backslash B_{r}(0)$ при любых $R>r>0$, $u \in \mathfrak{U}$, и получить оценку $q\left(e^{-a \tau} G_{k}^{\tau} u\right) \leqslant r(u)$, равномерную по $\tau \in \Sigma_{\theta-\delta} \backslash B_{r}(0)$. Поэтому внутри сектора $\Sigma_{\theta} U^{(k)}(\tau) u=G_{k}^{\tau} u, k \in \mathbb{N}, u \in \mathfrak{U}$.

Возьмем контур

$$
\Gamma_{1}=\left\{\nu \in \mathbb{C}: \nu=\varepsilon-|x|+i x \operatorname{tg}\left(\frac{\pi}{2}+\delta\right), x \in \mathbb{R}\right\} .
$$

Пусть $\xi=(p+1)^{-1} \tau, \tau \in \Sigma_{\theta-\delta}$. Построим контуры

$$
\Gamma_{\xi}^{k}=\left\{\mu_{k} \in \mathbb{C}: \xi\left(\mu_{k}-a\right)=\nu \in \Gamma_{1}\right\} \subset S_{a, \theta}^{L}(M) .
$$

Используя равенства

$$
\begin{aligned}
U(\tau)= & U(\xi(p+1))=(2 \pi i)^{-p-1} \int_{\Gamma_{\xi}^{0}} d \mu_{0} \cdots \int_{\Gamma_{\xi}^{p-1}} d \mu_{p-1} \\
& \times \int_{\Gamma_{\xi}^{p}} R_{(\mu, p)}^{L}(M) e^{\tau /(p+1) \sum_{k=0}^{p} \mu_{k}} d \mu_{p}, \\
U^{(n)}(\tau)= & (p+1)^{-n}(2 \pi i)^{-p-1} \sum_{k_{0}+k_{1}+\cdots+k_{p}=n} \frac{n !}{k_{0} ! k_{1} ! \cdots k_{p} !} \\
\times & \int_{\Gamma_{\xi}^{0}} \mu_{0}^{k_{0}} e^{\mu_{0} \xi} d \mu_{0} \cdots \int_{\Gamma_{\xi}^{p-1}} \mu_{p-1}^{k_{p-1}} e^{\mu_{p-1} \xi} d \mu_{p-1} \int_{\Gamma_{\xi}^{p}} R_{(\mu, p)}^{L}(M) \mu_{p}^{k_{p}} e^{\mu_{p} \xi} d \mu_{p}
\end{aligned}
$$

и стандартные рассуждения (см., например, $[2 ;$ гл. $1, \S 3])$, нетрудно показать эквинепрерьвность семейств $\left\{e^{-a \tau} \tau^{n} U^{(n)}(\tau): \tau \in \Sigma_{\theta-\delta} \cap B_{R}(0)\right\}, \delta>0, R>0$, $n \in\{0\} \cup \mathbb{N}$, и экспоненциальную ограниченность семейства $\left\{U(\tau): \tau \in \Sigma_{\theta-\delta}\right\}$.

Тот факт, что $\operatorname{im} U(\tau) \subset \operatorname{dom} M$ при всех $\tau \in \Sigma_{\theta}$, следует из замкнутости оператора $M$ и сходимости интеграла

$$
\int_{\Gamma} M R_{\mu}^{L}(M) e^{\mu \tau} u d \mu=\int_{\Gamma} \mu L R_{\mu}^{L}(M) e^{\mu \tau} u d \mu-\int_{\Gamma} L e^{\mu \tau} u d \mu=L G_{1}^{\tau} u .
$$


Итак, $M U(\tau) u=L G_{1}^{\tau} u=L U^{(1)}(\tau) u$, что и требовалось.

Далее, возьмем контур $\Gamma^{\prime}=\{\nu \in \mathbb{C}: \nu=\mu+1, \mu \in \Gamma\}, \tau, \sigma \in \Sigma_{\theta}, u \in \mathfrak{U}$ и, используя тождество (1.1), получим

$$
\begin{aligned}
U(\tau) U(\sigma) u= & \frac{1}{(2 \pi i)^{2}} \int_{\Gamma} R_{\mu}^{L}(M) e^{\mu \tau} d \mu \int_{\Gamma^{\prime}} R_{\nu}^{L}(M) u e^{\nu \sigma} d \nu \\
= & \frac{1}{(2 \pi i)^{2}} \int_{\Gamma} R_{\mu}^{L}(M) u e^{\mu \tau} d \mu \int_{\Gamma^{\prime}} \frac{e^{\nu \sigma}}{\nu-\mu} d \nu \\
& \quad-\frac{1}{(2 \pi i)^{2}} \int_{\Gamma^{\prime}} R_{\nu}^{L}(M) u e^{\nu \sigma} d \nu \int_{\Gamma} \frac{e^{\mu \tau}}{\nu-\mu} d \mu \\
= & \frac{1}{2 \pi i} \int_{\Gamma} R_{\mu}^{L}(M) u e^{\mu(\tau+\sigma)} d \mu=U(\tau+\sigma) u
\end{aligned}
$$

поскольку

$$
\int_{\Gamma^{\prime}} \frac{e^{\nu \sigma}}{\nu-\mu} d \nu=2 \pi i e^{\mu \sigma}, \quad \int_{\Gamma} \frac{e^{\mu \tau}}{\nu-\mu} d \mu=0
$$

СлЕДСТВИЕ 2.1. Если оператор $M(L, p)$-секториален с константой $a=0$, то әквинепрерывны семейства операторов

$$
\left\{\tau^{n} U^{(n)}(\tau): \tau \in \Sigma_{\theta-\delta}\right\}, \quad\left\{\tau^{n} F^{(n)}(\tau): \tau \in \Sigma_{\theta-\delta}\right\}, \quad n \in\{0\} \cup \mathbb{N} .
$$

СЛЕДСТВИЕ 2.2. Пусть пространства $\mathfrak{U}, \mathfrak{F}$ банаховы, а оператор $M(L, p)$ секториален. Тогда $\forall n \in \mathbb{N}, \forall T>0 \quad \exists C>0 \quad \forall t \in(0, T)$

$$
\left\|U^{(n)}(t)\right\| \leqslant \frac{C e^{a t}}{t^{n}}, \quad\left\|F^{(n)}(t)\right\| \leqslant \frac{C e^{a t}}{t^{n}} .
$$

При $n=0$ или $а=0$ оченка справедлива сразу для всех $t \in \mathbb{R}_{+}$.

ЗАмечАниЕ 2.1. В условиях теоремы 2.1 из (2.1), (1.2) имеем

$$
\begin{aligned}
& L U(t) u=F(t) L u \quad \forall u \in \mathfrak{U}, \quad \forall t \in \mathbb{R}_{+}, \\
& M U(t) u=F(t) M u \quad \forall u \in \operatorname{dom} M, \quad \forall t \in \mathbb{R}_{+} .
\end{aligned}
$$

\section{§3. Ядра и образы разрешающих полугрупп}

Лемма 3.1. Пусть оператор $M$ (L,p)-секториален. Тогда справедливо следующее: $\forall u \in \operatorname{im} R_{(\mu, p)}^{L}(M) \quad\left(\forall f \in \operatorname{im} L_{(\mu, p)}^{L}(M)\right) \quad \lim _{t \rightarrow 0+} U(t) u=u$ $\left(\lim _{t \rightarrow 0+} F(t) f=f\right)$.

ДокАЗАтЕльство. Возьмем вектор $u=\left(R_{\beta}^{L}(M)\right)^{p+1} v$. В силу леммы 1.2,i) мы можем выбрать точку $\beta \in \mathbb{R}$ справа от контура $\Gamma$, удовлетворяющего (2.2). Тогда в силу тождества (1.1)

$$
U(t) u=\left(R_{\beta}^{L}(M)\right)^{p}\left(\frac{1}{2 \pi i} \int_{\Gamma} \frac{R_{\mu}^{L}(M) v}{\beta-\mu} e^{\mu t} d \mu+R_{\beta}^{L}(M) \frac{1}{2 \pi i} \int_{\Gamma} \frac{e^{\mu t} v}{\mu-\beta} d \mu\right)
$$


причем последний интеграл равен нулю в силу выбора $\beta$. Из доказательства теоремы 2.1 следуют равномерная по $t \in(0, \delta)$ сходимость интеграла $U(t) u$ и возможность предельного перехода при $t \rightarrow 0+$ под знаком интеграла:

$$
\lim _{t \rightarrow 0+} U(t) u=\left(R_{\beta}^{L}(M)\right)^{p} \frac{1}{2 \pi i} \int_{\Gamma} \frac{R_{\mu}^{L}(M) v}{\beta-\mu} d \mu .
$$

При достаточно большом $R$ построим окружность $S_{R}(0)=\{\mu \in \mathbb{C}:|\mu|=R\}$. В качестве контура $\gamma_{R, 1}$ возьмем часть контура $\Gamma$, лежашую внутри $S_{R}(0)$, и правую часть окружности $S_{R}(0)$, отсекаемую контуром $\Gamma$, проходимую по часовой стрелке. Через $\gamma_{R, 2}$ обозначим эту же часть окружности, проходимую против часовой стрелки. Кроме того, $\gamma_{R, 3}$ - часть контура $\Gamma$, не попавшая внутрь $S_{R}(0)$. Нетрудно показать, что для любой непрерывной полунормы $q(\cdot)$ на пространстве $\mathfrak{U}$ при $R \rightarrow \infty$

$$
q\left(\left(R_{\beta}^{L}(M)\right)^{p} \int_{\gamma_{R, k}} \frac{R_{\mu}^{L}(M) v}{\beta-\mu} d \mu\right) \rightarrow 0, \quad k=2,3 .
$$

Отсюда, так как точка $\beta$ лежит внутри контура $\gamma_{R, 1}$,

$$
\begin{aligned}
& \lim _{t \rightarrow 0+} U(t) u=\frac{1}{2 \pi i}\left(R_{\beta}^{L}(M)\right)^{p} \sum_{k=1}^{3} \int_{\gamma_{R, k}} \frac{R_{\mu}^{L}(M) v}{\beta-\mu} d \mu \\
& =\lim _{R \rightarrow+\infty} \frac{1}{2 \pi i}\left(R_{\beta}^{L}(M)\right)^{p} \sum_{k=1}^{3} \int_{\gamma_{R, k}} \frac{R_{\mu}^{L}(M) v}{\beta-\mu} d \mu=\left(R_{\beta}^{L}(M)\right)^{p+1} v=u
\end{aligned}
$$

ЗАмЕчАниЕ 3.1. Если $\{V(\tau): \tau \in \Sigma\}$ - сильно голоморфная полугруппа, то, используя теорему единственности аналитической функции, нетрудно показать, что при всех $\tau_{1}, \tau_{2} \in \Sigma \operatorname{ker} V\left(\tau_{1}\right)=\operatorname{ker} V\left(\tau_{2}\right)$.

ОПРЕДЕЛЕНИЕ 3.1. Ядром голоморфной полугруппь $\{V(\tau): \tau \in \Sigma\}$ будем называть множество $\operatorname{ker} V(\cdot)=\operatorname{ker} V(\tau), \tau \in \Sigma$.

В силу предыдушего замечания это определение корректно. Обозначим через $\widehat{L}_{0}\left(\widehat{M}_{0}\right)$ сужение оператора $L(M)$ на $\operatorname{ker} U(\cdot)(\operatorname{ker} U(\cdot) \cap \operatorname{dom} M)$. Из замечания 2.1 сразу следует

Лемма 3.2. Пусть оператор $M(L, p)$-секториален. Тогда

$$
\widehat{L}_{0} \in \mathscr{L}(\operatorname{ker} U(\cdot) ; \operatorname{ker} F(\cdot)), \quad \widehat{M}_{0}: \operatorname{ker} U(\cdot) \cap \operatorname{dom} M \rightarrow \operatorname{ker} F(\cdot) .
$$

Лемма 3.3. Пусть оператор $M(L, p)$-секториален. Тогда $\sigma^{\widehat{L}_{0}}\left(\widehat{M}_{0}\right)=\varnothing$.

ДокАЗАТЕльство. Непосредственно доказывается, что при $t \in \mathbb{R}_{+}$

$$
\frac{1}{2 \pi i} \int_{\Gamma} \frac{(\mu L-M)^{-1} e^{(\mu-\lambda) t}}{\mu-\lambda} d \mu=(\lambda L-M)^{-1}
$$

если контур Г, удовлетворяюший (2.2), выбрать лежашим “правее” точки $\lambda$. 
Tеорема 3.1. Пусть оператор $M(L, p)$-секториален. Тогда $\operatorname{ker} U(\cdot)=\mathfrak{U}^{0}$, $\operatorname{ker} F(\cdot)=\mathfrak{F}^{0}$.

ДокаЗАТЕльСтво. Возьмем $\varphi_{q} \in \mathfrak{U}^{0} \backslash\{0\}$, т.е. $\varphi_{q}$ - собственный или $M$-присоединенньй вектор высоты $q \leqslant p$ оператора $L$. Понятно, что собственный вектор согласно (2.1) принадлежит $\operatorname{ker} U(\cdot)$. Для $M$-присоединенного вектора $\varphi_{q}$ имеем то же самое согласно лемме 1.1. Таким образом, $\mathfrak{U}^{0} \subset \operatorname{ker} U(\cdot)$.

Докажем обратное включение. Рассмотрим вектор $\psi=R_{(\mu, p)}^{L}(M) \varphi$, где $\varphi \in$ $\operatorname{ker} U(\cdot)$. В силу лемм $3.2,3.3 \psi=R_{(\mu, p)}^{\widehat{L}_{0}}\left(\widehat{M}_{0}\right) \varphi \in \operatorname{ker} U(\cdot)$, поэтому по лемме 3.1 $0=\lim _{t \rightarrow 0+} U(t) \psi=\psi=R_{(\mu, p)}^{L}(M) \varphi$. Таким образом, вектор $\varphi \in \mathfrak{U}^{0}$. Следовательно, $\operatorname{ker} U(\cdot)=\mathfrak{U}^{0}$.

Теперь возьмем $f \in \mathfrak{F}^{0}$. В силу леммы 1.2 ,ii) $f=M \varphi$, где $\varphi \in \mathfrak{U}^{0} \cap \operatorname{dom} M$. Поэтому для любого $t \in \mathbb{R}_{+} F(t) f=F(t) M \varphi=M U(t) \varphi=M 0=0$ по доказанному. Таким образом, $\mathfrak{F}^{0} \subset \operatorname{ker} F(\cdot)$.

Пусть $f \in \operatorname{ker} F(\cdot)$, тогда $\widehat{M}_{0}^{-1} f=\varphi \in \operatorname{ker} U(\cdot)=\mathfrak{U}^{0}$. Поэтому

$$
L_{(\mu, p)}^{L}(M) f=L_{(\mu, p)}^{L}(M) \widehat{M}_{0} \varphi=M R_{(\mu, p)}^{L}(M) \varphi=0 .
$$

СлЕДСтвИЕ 3.1. Пусть оператор $M(L, p)$-секториален. Тогда

i) существует оператор $M_{0}^{-1} \in \mathscr{L}\left(\mathfrak{F}^{0} ; \mathfrak{U}^{0}\right)$;

ii) операторы $H=M_{0}^{-1} L_{0}, G=M_{0}^{-1} L_{0}$ нильпотентны степени не более $p$.

ДокаЗАТЕЛЬство. Утверждение і) следует из леммы 3.3 и теоремы 3.1 $\left(0 \in \rho^{L_{0}}\left(M_{0}\right)\right)$, утверждение ii) - из предыдушего и из леммы $\left.1.3, \mathrm{i}\right)$.

ОПРеДЕЛЕНИЕ 3.2. Образом полугруппь $\left\{V(t) \in \mathscr{L}(\mathfrak{V}): t \in \mathbb{R}_{+}\right\}$назовем множество $\operatorname{im} V(\cdot)=\left\{v \in \mathfrak{V}: v=\lim _{t \rightarrow 0+} V(t) v\right\}$.

ЛЕмма 3.4. Для сильно голоморфной полугруппы $\{V(t): t \in \Sigma\}$ имеем $\operatorname{ker} V(\cdot) \cap \operatorname{im} V(\cdot)=\{0\}$.

ДокаЗАТЕЛЬСтво. Пусть $v \in \operatorname{ker} V(\cdot) \cap \operatorname{im} V(\cdot)$. Тогда в силу замечания 3.1 $\forall t \in \mathbb{R}_{+} V(t) v=0$. Поэтому $v=\lim _{t \rightarrow 0+} V(t) v=0$.

Лемма 3.5. Пусть полугруппа $\left\{V(t) \in \mathfrak{V}: t \in \mathbb{R}_{+}\right\}$сильно непрерьвна и экспоненииально ограничена. Тогда $\operatorname{im} V(\cdot)=\overline{\bigcup_{t>0}} \operatorname{im} V(t)$.

ДокАЗАТЕЛЬСтво. Докажем замкнутость образа полугруппы $\operatorname{im} V(\cdot)$. Возьмем обобшенную последовательность $\left\{v_{\alpha}\right\} \subset \operatorname{im} V(\cdot)$, сходящуюся к вектору $v \in \mathfrak{V}$. Тогда для любой непрерывной на $\mathfrak{V}$ полунормы $q(\cdot)$ сушествует непрерывная на $\mathfrak{V}$ полунорма $r(\cdot)$ такая, что

$$
\begin{aligned}
\lim _{t \rightarrow 0+} q(V(t) v-v) & =\lim _{t \rightarrow 0+} q\left(V(t) v-V(t) v_{\alpha}+V(t) v_{\alpha}-v_{\alpha}+v_{\alpha}-v\right) \\
& \leqslant \lim _{t \rightarrow 0+} e^{a t} r\left(v-v_{\alpha}\right)+\lim _{t \rightarrow 0+} q\left(V(t) v_{\alpha}-v_{\alpha}\right)+q\left(v-v_{\alpha}\right) \\
& =r\left(v-v_{\alpha}\right)+q\left(v-v_{\alpha}\right) .
\end{aligned}
$$

Переходим к пределу по $\alpha$, получаем, что $v \in \operatorname{im} V(\cdot)$. 
Вследствие сильной непрерывности полугруппы очевидно, что для всех $s \in \mathbb{R}_{+}$ $\operatorname{im} V(s) \subset \operatorname{im} V(\cdot)$. В силу замкнутости $\operatorname{im} V(\cdot)$ имеем $\overline{\bigcup_{s>0} \operatorname{im} V(s)} \subset \operatorname{im} V(\cdot)$. Возьмем $v \in \operatorname{im} V(\cdot)$. Тогда последовательность

$$
\left\{v_{k}=V\left(\frac{1}{k}\right) v\right\}_{k=1}^{\infty} \subset \bigcup_{s>0} \operatorname{im} V(s)
$$

такова, что $v_{k} \rightarrow v$ при $k \rightarrow \infty$, поэтому $v \in \overline{\bigcup_{s>0} \operatorname{im} V(s)}$.

Tеорема 3.2. Пусть оператор $M(L, p)$-секториален. Тогда $\operatorname{im} U(\cdot)=\mathfrak{U}^{1}$, $\operatorname{im} F(\cdot)=\mathfrak{F}^{1}$.

ДокаЗАтЕльство. Согласно лемме $3.1 \mathrm{im} R_{(\mu, p)}^{L}(M) \subset \operatorname{im} U(\cdot)$. А в силу замкнутости $\operatorname{im} U(\cdot)$ имеем $\mathfrak{U}^{1} \subset \operatorname{im} U(\cdot)$.

В соответствии с теоремой Коши и тождеством (1.1)

$$
\begin{aligned}
U(t) u & =\frac{1}{2 \pi i} \int_{\Gamma} R_{\lambda}^{L}(M) e^{\lambda t} u d \lambda \\
& =\frac{1}{2 \pi i} \int_{\Gamma} R_{\lambda}^{L}(M) e^{\lambda t} u d \lambda-\frac{1}{2 \pi i} \int_{\Gamma} R_{\mu_{0}}^{L}(M) e^{\lambda t} u d \lambda \\
& =\frac{1}{2 \pi i} R_{\mu_{0}}^{L}(M) \int_{\Gamma}\left(\mu_{0}-\lambda\right) R_{\lambda}^{L}(M) e^{\lambda t} u d \lambda .
\end{aligned}
$$

Повторив эту процедуру $p$ раз, для $\mu_{0}, \mu_{1}, \ldots, \mu_{p} \in \rho^{L}(M)$ получим

$$
U(t) u=R_{(\mu, p)}^{L}(M) \frac{1}{2 \pi i} \int_{\Gamma} \prod_{k=0}^{p}\left(\mu_{k}-\lambda\right) R_{\lambda}^{L}(M) e^{\lambda t} u d \lambda .
$$

Таким образом, в силу леммы $3.5 \mathrm{im} U(\cdot) \subset \mathfrak{U}^{1}$.

Обозначим

$$
\widetilde{U}(t)=\left.U(t)\right|_{\tilde{\mathfrak{U}}}, \quad \widetilde{F}(t)=\left.F(t)\right|_{\widetilde{\mathfrak{F}}} .
$$

Лемма 3.6. Пусть оператор $M(L, p)$-секториален. Тогда

$$
\widetilde{P}=\operatorname{slim}_{t \rightarrow 0+} \widetilde{U}(t), \quad \widetilde{Q}=\operatorname{sim}_{t \rightarrow 0+} \widetilde{F}(t)
$$

ДокАЗАТЕльство. Проекторы $\widetilde{P}$ и $\widetilde{Q}$ определены ранее в лемме 1.4, ii). Утверждение данной леммы следует из теорем $3.1,3.2$.

ЗАмЕчАНИЕ 3.2. Из леммы 3.6 следует, что при условии $(L, p)$-секториальности оператора $M$ полугрупा $\left\{\widetilde{U}(t): t \in \mathbb{R}_{+}\right\},\left\{\widetilde{F}(t): t \in \mathbb{R}_{+}\right\}$можно доопределить в нуле по непрерывности: $\widetilde{U}(0)=\widetilde{P}, \widetilde{F}(0)=\widetilde{Q}$. 


\section{§4. Фазовые пространства}

Пусть $\mathfrak{U}$ - секвенциально полное локально выпуклое пространство, функции $u: \mathbb{R} \rightarrow \mathfrak{U}, w: \mathbb{R} \rightarrow \mathbb{C}$. Их сверткой называется интеграл Римана

$$
\int_{\mathbb{R}} u(s) w(t-s) d s=u * w: \mathbb{R} \rightarrow \mathfrak{U} .
$$

Сразу отметим симмметричность свертки $u * w=\int_{\mathbb{R}} w(s) u(t-s) d s=w * u$.

Символом $D^{k}$ будем обозначать $k$-ю производную функции, нижний индекс “нуль" при обозначении функционального пространства будет обозначать финитность входящих в него функций.

Лемма 4.1. Пусть $и \in C_{0}^{n}(\mathbb{R} ; \mathfrak{U}), w \in C_{0}^{m}(\mathbb{R} ; \mathbb{C})$. Тогда вектор-функиия $u * w \in C_{0}^{m+n}(\mathbb{R} ; \mathfrak{U})$, причем

$$
\begin{aligned}
D^{k}(u * w)=u * D^{k} w, & k \leqslant m \in\{0\} \cup \mathbb{N}, \\
D^{l}(u * w)=D^{l} u * w, & l \leqslant n \in\{0\} \cup \mathbb{N} .
\end{aligned}
$$

Следуя [13; гл. XVII, §4], аппроксимативной единицей при $\alpha \rightarrow \alpha_{0}$ будемназывать семейство неотрицательных функций $\left\{\Delta_{\alpha}: \mathbb{R} \rightarrow \mathbb{R} ; \alpha \in \mathscr{A}\right\}$ таких, что, во-первых, при любом $\alpha \in \mathscr{A} \int_{\mathbb{R}} \Delta_{\alpha}(s) d s=1$, а во-вторых, для любой окрестности $\mathscr{O}$ нуля $\lim _{\alpha \rightarrow \alpha_{0}} \int_{\mathscr{O}} \Delta_{\alpha}(s) d s=1$.

ЛЕмма 4.2. Пусть функиия $u: \mathbb{R} \rightarrow \mathfrak{U}$ ограничена, $\mathscr{A} \subset \mathbb{R}, \alpha_{0} \in \overline{\mathscr{A}}$, $\left\{\Delta_{\alpha}: \mathbb{R} \rightarrow \mathbb{R} ; \alpha \in \mathscr{A}\right\}$ - аппроксимативная единииа при $\alpha \rightarrow \alpha_{0}$. Если при любом $\alpha \in \mathscr{A}$ свертка $u * \Delta_{\alpha}$ существует, а функция и непрерывна на $(a, b)$, то $и * \Delta_{\alpha}(t)$ поточечно сходится $к u(t)$ на $(a, b)$ при $\alpha \rightarrow \alpha_{0}$.

Леммы 4.1 и 4.2 доказываются стандартным образом.

Лемма 4.3. і) Если функиия $и \in C([0, T] ; \mathfrak{U}) \cap C^{1}((0, T] ; \mathfrak{U})$ удовлетворяет уравнению (0.2) на интервале $(0, T]$, то при $\mu \in \rho^{L}(M)$

$$
\int_{0}^{t} e^{-\mu \tau} u(\tau) d \tau=R_{\mu}^{L}(M)\left(u(0)-e^{-\mu t} u(t)\right)
$$

ii) если функиия $f \in C([0, T] ; \mathfrak{F}) \cap C^{1}((0, T] ; \mathfrak{F})$ удовлетворяет уравнению (0.3) на интервале $(0, T]$, то при $\mu \in \rho^{L}(M)$

$$
\int_{0}^{t} e^{-\mu \tau} f(\tau) d \tau=L_{\mu}^{L}(M)\left(f(0)-e^{-\mu t} f(t)\right)
$$

ДокАЗАТЕЛЬство. Интегрируя по частям, получаем

$$
\mu L \int_{0}^{t} e^{-\mu \tau} u(\tau) d \tau=-e^{-\mu t} L u(t)+L u(0)+M \int_{0}^{t} e^{-\mu \tau} u(\tau) d \tau .
$$

Если $\mu \in \rho^{L}(M)$, то имеем тождество (4.1). Выбирая в нем $u(t)=(\alpha L-M)^{-1} f(t)$, $\alpha \in \rho^{L}(M)$, и используя следуюшее из (1.1) тождество $(\mu L-M)^{-1} L_{\alpha}^{L}(M)=$ $(\alpha L-M)^{-1} L_{\mu}^{L}(M)$, получаем (4.2). 
Лемма 4.4 [14]. Пусть $\varphi \in L_{1}((0, T) ; \mathfrak{U})$ и для любой непрерывной в пространстве $\mathfrak{U}$ полунормы $q(\cdot)$

$$
\varlimsup_{\mu \rightarrow+\infty} \frac{1}{\mu} \ln q\left(\int_{0}^{T} e^{\mu s} \varphi(s) d s\right) \leqslant h<T .
$$

Тогда $\varphi(s)=0$ почти всюду на $[h, T]$.

TеОРема 4.1. Пусть $T_{1}, T_{2} \in \mathbb{R}$. Тогда для любой функиии $u \in C^{1}\left(\left(T_{1}, T_{2}\right) ; \mathfrak{U}\right)$ $\left(f \in C^{1}\left(\left(T_{1}, T_{2}\right) ; \mathfrak{F}\right)\right)$, удовлетворяющей уравнению $(0.2)((0.3))$ на интервале $\left(T_{1}, T_{2}\right)$, имеем при $t \in\left(T_{1}, T_{2}\right)$

$$
u(t) \in \overline{\bigcap_{s=0}^{\infty} \operatorname{im} R_{(\mu, s)}^{L}(M)} \quad\left(f(t) \in \overline{\bigcap_{s=0}^{\infty} \operatorname{im} L_{(\mu, s)}^{L}(M)}\right) .
$$

ДокаЗАТЕЛЬСтво. Пусть $u \in C^{\infty}\left(\left(T_{1}, T_{2}\right) ; \mathfrak{U}\right)$ - решение уравнения $(0.2)$. Тогда $R_{\alpha}^{L}(M) \dot{u}=(\alpha L-M)^{-1} M u=\alpha R_{\alpha}^{L}(M) u-u$. Отсюда

$$
u=R_{\alpha}^{L}(M)\left(\alpha-\frac{d}{d t}\right) u
$$

По индукции легко доказать, что при $k \in \mathbb{N}$

$$
u=\left(R_{\alpha}^{L}(M)\right)^{k} \sum_{m=0}^{k}(-1)^{m} C_{k}^{m} \alpha^{k-m} \frac{d^{m}}{d t^{m}} u=\left(R_{\alpha}^{L}(M)\right)^{k}\left(\alpha-\frac{d}{d t}\right)^{k} u .
$$

Следовательно, $u(t) \in \bigcap_{s=0}^{\infty} \operatorname{im} R_{(\mu, s)}^{L}(M) \forall t \in\left(T_{1}, T_{2}\right)$.

Рассмотрим функцию $\varphi \in C_{0}^{\infty}(\mathbb{R} ; \mathbb{R})$, равную $k e^{-\left(1-t^{2}\right)^{-1}}$ при $|t|<1$ и нулю при $|t| \geqslant 1$. Здесь $k=\left(\int_{-1}^{1} e^{-\left(1-s^{2}\right)^{-1}} d s\right)^{-1}$. Нетрудно убедиться, что $\left\{\Delta_{\alpha}=\right.$ $\left.\alpha^{-1} \varphi\left(\alpha^{-1} t\right): \alpha>0\right\}-$ аппроксимативная единица при $\alpha \rightarrow 0+$.

Доопределим решение $u \in C^{1}\left(\left(T_{1}, T_{2}\right) ; \mathfrak{U}\right)$ на всю числовую прямую, положив $u(t)=0, t \notin\left[T_{1}+\varepsilon, T_{2}-\varepsilon\right], \varepsilon>0$. Возьмем $T>0$ и достаточно малое $\alpha$ так, чтобы $\Delta_{\alpha}(s)=0$ при $|s| \geqslant T$. Покажем, что свертки доопределенного решения уравнения $(0.2)$ с функциями $\Delta_{\alpha}$ будут решениями уравнения (0.2). Действительно,

$$
\begin{aligned}
L\left(u * \Delta_{\alpha}\right)_{t}= & L \frac{d}{d t} \int_{T_{1}-T}^{T_{2}+T} u(s) \Delta_{\alpha}(t-s) d s=L \frac{d}{d t} \int_{t-T_{2}-T}^{t-T_{1}+T} \Delta_{\alpha}(s) u(t-s) d s \\
= & L \Delta_{\alpha}\left(t-T_{1}+T\right) u\left(T_{1}-T\right)-L \Delta_{\alpha}\left(t-T_{2}-T\right) u\left(T_{2}+T\right) \\
& \quad+L \int_{t-T_{2}-T}^{t-T_{1}+T} \Delta_{\alpha}(s) \frac{d}{d t} u(t-s) d s \\
= & \int_{T_{1}-T}^{T_{2}+T} M u(s) \Delta_{\alpha}(t-s) d s=M\left(u * \Delta_{\alpha}\right), \quad t \in \mathbb{R} .
\end{aligned}
$$

Все указанные свертки существуют по лемме 4.1 и в силу приведенных тождеств. Согласно лемме $4.1 u * \Delta_{\alpha} \in C_{0}^{\infty}(\mathbb{R} ; \mathfrak{U})$. Доопределенные нулем решения $u$ ограничены и непрерывны на $\left[T_{1}+\varepsilon, T_{2}-\varepsilon\right]$, поэтому согласно лемме 4.2 получаем, что 
бесконечно дифференцируемые решения $u * \Delta_{\alpha}$ на интервале $\left(T_{1}+\varepsilon, T_{2}-\varepsilon\right)$ поточечно стремятся к один раз непрерывно дифференцируемому решению $u$ при $\alpha \rightarrow 0+$. Поэтому для решений из класса $C^{1}\left(\left(T_{1}, T_{2}\right) ; \mathfrak{U}\right)$ имеем $u \in \overline{\bigcap_{s=0}^{\infty} \operatorname{im} R_{(\mu, s)}^{L}(M)}$ при $t \in\left(T_{1}, T_{2}\right)$ в силу произвольности $\varepsilon$.

Следуя [5; гл. I, §3, определение 3.1], ослабленным решением уравнения (0.2) будем называть функцию $u \in C^{1}\left(\mathbb{R}_{+} ; \mathfrak{U}\right) \cap C\left(\overline{\mathbb{R}}_{+} ; \mathfrak{U}\right)$, удовлетворяюшую уравнению при $t \in \mathbb{R}_{+}$. Ослабленной задачей Коши для уравнения (0.2) будем назьвать задачу нахождения ослабленного решения уравнения, удовлетворяюшего условию $u(0)=u_{0}$.

ОПРЕДЕЛЕНИЕ 4.1. Замкнутое множество $\mathfrak{P} \subset \mathfrak{U}$ называется фазовым пространством уравнения (0.2), если

i) любое ослабленное решение $u=u(t)$ уравнения $(0.2)$ лежит в $\mathfrak{P}$, т.е. $u(t) \in \mathfrak{P}$ $\forall t \in \overline{\mathbb{R}}_{+}$;

ii) для любого $u_{0} \in \mathfrak{P}$ существует единственное решение ослабленной задачи Коши $u(0)=u_{0}$ для уравнения $(0.2)$.

Tеорема 4.2. Пусть оператор $M$ (L,p)-секториален. Тогда $\mathfrak{U}^{1}\left(\mathfrak{F}^{1}\right)$ есть фазовое пространство уравнения (0.2) ((0.3)).

ДоКАЗАТЕЛЬСТво. Очевидны включения

$$
\overline{\bigcap_{s=0}^{\infty} \operatorname{im} R_{(\mu, s)}^{L}(M)} \subset \overline{\bigcap_{s=0}^{\infty} \overline{\operatorname{im} R_{(\mu, s)}^{L}(M)}}=\bigcap_{s=0}^{\infty} \overline{\operatorname{im} R_{(\mu, s)}^{L}(M)} \subset \mathfrak{U}^{1} .
$$

Возьмем в предыдушей теореме $T_{1}=0$. Тогда для ослабленных решений $u$ имеем $u(t) \in \mathfrak{U}^{1}$ при всех $t \in \mathbb{R}_{+}$в силу теоремы 4.1. Принадлежность точки $u(0)$ множеству $\mathfrak{U}^{1}$ следует из его замкнутости и непрерывности ослабленных решений в точке $t=0$.

Установим единственность решения ослабленной задачи Коши. Из (4.1) для ослабленного решения уравнения (0.2), удовлетворяюшего условию $u(0)=0$, при $t=T>0$ получим

$$
R_{\mu}^{L}(M) u(T)=\int_{0}^{T} e^{\mu(T-\tau)} u(\tau) d \tau=\int_{0}^{T} e^{\mu s} u(T-s) d s .
$$

Согласно лемме 1.6 для $\varphi(s)=u(T-s)$ и любой непрерывной в $\mathfrak{U}$ полунормы $q(\cdot)$ существует непрерьвная на $\mathfrak{F}$ полунорма $r(\cdot)$ такая, что

$$
\varlimsup_{\mu \rightarrow+\infty} \frac{1}{\mu} \ln q\left(\int_{0}^{T} e^{\mu s} \varphi(s) d s\right) \leqslant \varlimsup_{\mu \rightarrow+\infty} \frac{\ln \mu^{p+1}+\ln r(L u(T))}{\mu}=0 .
$$

Отсюда по лемме $4.4 u(t)=0$ почти всюду на $[0, T]$. А так как $u$-непрерывная функция, а $T$ произвольно, то $u(t)=0$ при всех $t \in \overline{\mathbb{R}}_{+}$.

Сушествование решения ослабленной задачи Коши для уравнения (0.2) при $u_{0} \in \mathfrak{U}^{1}$ следует из теорем 2.1,3.2.

ЗАмЕчАнИЕ 4.1. Из единствености решения ослабленной задачи Коши следует единственность разрешаюшей полугруппы. 
СлЕДСтвИЕ 4.1. Пусть оператор $M$ (L,p)-секториален. Тогда любое ослабленное решение уравнения (0.2) или уравнения (0.3) имеет голоморфное продолжение в сектор $\Sigma_{\theta}=\{\tau \in \mathbb{C}:|\arg \tau|<\theta-\pi / 2\}$.

ДокАЗАТЕЛЬСтво. Любое ослабленное решение $u(t)$ уравнения $(0.2)$ в силу его непрерывности вплоть до нуля является решением ослабленной задачи Коши с начальным значением $u(0)$, которое согласно теореме 4.2 принадлежит $\mathfrak{U}^{1}$. По той же теореме решение ослабленной задачи Коши единственно, поэтому задается полугруппой (2.1), которая сильно голоморфна в секторе $\Sigma_{\theta}$.

\section{§5. Единицы разрешающих полугрупп}

TEOРема 5.1. Пусть пространство $\mathfrak{U}$ (F) полурефлексивно, оператор $M$ (L, p)-секториален. Тогда $\mathfrak{U}=\mathfrak{U}^{0} \oplus \mathfrak{U}^{1} \quad\left(\mathfrak{F}=\mathfrak{F}^{0} \oplus \mathfrak{F}^{1}\right)$.

ДокАЗАТЕЛЬСТво. Возьмем произвольньй вектор $u \in \mathfrak{U}$. Из теоремы 2.1 следует, что для любой непрерывной полунормы $q(\cdot)$ сушествует полунорма $r(\cdot$ ) такая, что сразу для всех $t \in(0,1) q(U(t) u) \leqslant r(u)$. Таким образом, множество $\mathscr{A}_{u}=\{U(1 / n) u: n \in \mathbb{N}\} \subset \mathfrak{U}$ ограничено. Из полурефлексивности пространства $\mathfrak{U}$ следует, что $\mathscr{A}_{u}$ относительно слабо компактно [1; приложение к гл. V, $\S 3$, теорема 1]. Поэтому из последовательности $\mathscr{A}_{u} \subset \mathfrak{U}^{1}$ мы можем выбрать подпоследовательность $\left\{U\left(1 / n_{k}\right) u\right\}$, слабо сходящуюся к некоторому вектору $v \in \mathfrak{U}$. Из замкнутости линейного подпространства $\mathfrak{U}^{1}$ следует его слабая замкнутость, поэтому $v \in \mathfrak{U}^{1}$.

Пусть $v_{k}=U\left(1 / n_{k}\right) u-v$, тогда

$$
\underset{k \rightarrow \infty}{\mathrm{w}-\lim } v_{k}=0 .
$$

По лемме 3.1

$$
\begin{aligned}
\underset{k \rightarrow \infty}{\mathrm{S}-\lim _{\mu}\left(R_{\mu}^{L}(M)\right)^{p+1} v_{k}} & =\underset{k \rightarrow \infty}{\mathrm{s}-\lim } U\left(\frac{1}{n_{k}}\right)\left(R_{\mu}^{L}(M)\right)^{p+1} u-\left(R_{\mu}^{L}(M)\right)^{p+1} v \\
& =\left(R_{\mu}^{L}(M)\right)^{p+1}(u-v) .
\end{aligned}
$$

Для любого элемента $u^{\prime}$ сопряженного к $\mathfrak{U}$ пространства $\mathfrak{U}^{\prime}$

$$
u^{\prime}\left(\left(R_{\mu}^{L}(M)\right)^{p+1} v_{k}\right)=v^{\prime}\left(v_{k}\right)
$$

где $v^{\prime} \in \mathfrak{U}^{\prime}$. Отсюда и в силу $(5.1),(5.2)$

$$
\underset{k \rightarrow \infty}{\mathrm{w}-\lim }\left(R_{\mu}^{L}(M)\right)^{p+1} v_{k}=0=\left(R_{\mu}^{L}(M)\right)^{p+1}(u-v),
$$

значит, $u-v \in \mathfrak{U}^{0}$. Таким образом, для произвольного $u \in \mathfrak{U}$ имеем $u=z+v$, где $z=u-v \in \mathfrak{U}^{0}, v \in \mathfrak{U}^{1}$.

Единицей сильно непрерывной полугруппь операторов $\left\{V(t): t \in \mathbb{R}_{+}\right\}$называется оператор s-lim $t \rightarrow 0+V(t) \in \mathscr{L}(\mathfrak{V})$, если он существует. Понятно, что единица полугруппы является проектором. 
ЗАмечаниЕ 5.1. Согласно теореме 5.1 и лемме 3.6 получаем, что в случае полурефиексивного пространства построенная на нем в теореме 2.1 полугруппа имеет единицу.

Чтобы получить существование единиц полугрупп операторов (2.1) без требования полуреф̆лексивности пространств, приходится усиливать условия на операторы $L, M$. Для этого вводится следуюшее определение.

ОПРЕДЕЛЕНИЕ 5.1. Оператор $M$ называется сильно $(L, p)$-секториальныцм справа $\left(\right.$ слева), если он $(L, p)$-секториален ипри любых $\lambda, \mu_{0}, \mu_{1}, \ldots, \mu_{p} \in S_{a, \theta}^{L}(M)$ справедливо следуюшее: для любого $u \in \operatorname{dom} M$ и для любой непрерывной в $\mathfrak{U}$ полунормы $r(\cdot)$ существует константа $c_{1}$, зависящая от $u$, такая, что

$$
r\left((\lambda-a) \prod_{k=0}^{p}\left(\mu_{k}-a\right) R_{(\mu, p)}^{L}(M)(\lambda L-M)^{-1} M u\right) \leqslant c_{1}(u),
$$

соответственно для всех $f$ из некоторого плотного в $\mathfrak{F}$ линеала $\stackrel{\circ}{\mathfrak{F}}$ и для любой непрерывной в $\mathfrak{F}$ полунормы $r(\cdot)$ сушествует константа $c_{2}$, зависяшая от $f$, такая, что

$$
r\left((\lambda-a) \prod_{k=0}^{p}\left(\mu_{k}-a\right) M(\lambda L-M)^{-1} L_{(\mu, p)}^{L}(M) f\right) \leqslant c_{2}(f) .
$$

Теорема 5.2. Пусть оператор $M$ сильно (L,p)-секториален справа (слева). Тогда существует единица полугруппы $\left\{U(t): t \in \mathbb{R}_{+}\right\} \quad\left(\left\{F(t): t \in \mathbb{R}_{+}\right\}\right)$.

ДокАЗАТЕЛЬСТво. Пусть $u \in \operatorname{dom} M, s>t>0, \tau=t /(p+1)$. Тогда

$$
\begin{aligned}
U(s) u-U(t) u= & U(t) \frac{1}{2 \pi i} \int_{\Gamma}\left(R_{\lambda}^{L}(M)-\frac{I}{\lambda}\right) u e^{\lambda(s-t)} d \lambda \\
= & (2 \pi i)^{-p-2} \int_{\Gamma_{0}} \cdots \int_{\Gamma_{p}} \int_{\Gamma} R_{(\mu, p)}^{L}(M)(\lambda L-M)^{-1} M u \\
& \times \exp \left(\sum_{k=0}^{p} \mu_{k} \tau+\lambda(s-t)\right) \frac{d \lambda}{\lambda} d \mu_{0} \cdots d \mu_{p},
\end{aligned}
$$

где контуры $\Gamma, \Gamma_{k}, k=\overline{0, p}$, удовлетворяют (2.2) и расположены в комплексных плоскостях переменных $\lambda, \mu_{k}, k=\overline{0, p}$, соответственно. Сделаем замены $\lambda=a+\nu(s-t)^{-1}, \mu_{k}=a+\nu_{k} / \tau, k=\overline{0, p}$. Очевидно, новые контуры интегрирования можно выбрать не зависящими от $s, t$ и $\tau$ так, чтобы все они имели вид (2.3). Обозначим эти контуры теми же символами $\Gamma, \Gamma_{0}, \Gamma_{1}, \ldots, \Gamma_{p}$. Из сильной $(L, p)$-секториальности оператора $M$ при любой полунорме $q(\cdot)$ имеем

$$
\begin{aligned}
q(U(s) u & -U(t) u) \leqslant(s-t)(2 \pi)^{-p-2} \int_{\Gamma_{0}} \ldots \int_{\Gamma_{p}} \int_{\Gamma}\left|\exp \left(\sum_{k=0}^{p} \nu_{k}+\nu+a s\right)\right| \\
& \times q\left(\prod_{k=0}^{p}\left(\left(\left(\nu_{k}+a \tau\right) L-\tau M\right)^{-1} L\right)((\nu+a(s-t)) L-(s-t) M)^{-1} M u\right) \\
& \times \frac{|d \nu|}{|\nu+a(s-t)|}\left|d \nu_{0}\right| \cdots\left|d \nu_{p}\right| \\
\leqslant & e^{a s}(s-t) c_{1}(u)\left(\int_{\Gamma_{0}} \frac{e^{\operatorname{Re} \nu_{0}}\left|d \nu_{0}\right|}{\left|\nu_{0}\right|}\right)^{p+1} \int_{\Gamma} \frac{e^{\operatorname{Re} \nu}|d \nu|}{|\nu||\nu-\delta|}
\end{aligned}
$$


при $s-t<\delta|a|^{-1}, \delta<\varepsilon($ из (2.3)). Отсюда

$$
q(U(s) u-U(t) u) \leqslant e^{a s}(s-t) c_{3}(u) \quad \forall u \in \operatorname{dom} M .
$$

Семейство операторов $\{U(t): t \in(0, \delta)\}$ эквинепрерывно в силу экспоненциальной ограниченности полугруппы. Учитывая секвенциальную полноту пространства $\mathfrak{U}$ и плотность множества $\operatorname{dom} M$ в $\mathfrak{U}$, из (5.3) получаем сушествование предела $P=$ $\mathrm{s}-\lim _{t \rightarrow 0+} U(t) \in \mathscr{L}(\mathfrak{U})$.

СЛЕДСТВИЕ 5.1. Пусть оператор $M$ сильно $(L, p)$-секториален справа (слева). Тогда $\mathfrak{U}=\mathfrak{U}^{0} \oplus \mathfrak{U}^{1} \quad\left(\mathfrak{F}=\mathfrak{F}^{0} \oplus \mathfrak{F}^{1}\right)$.

ДокАЗАТЕЛЬСтво. Проектором, расшепляющим пространство, является единица соответстствующей полугруппы.

Итак, если оператор $M$ сильно $(L, p)$-секториален справа (слева), либо если он $(L, p)$-секториален, а пространство $\mathfrak{U}(\mathfrak{F})$ полурефлексивно, то в силу лемм $1.4, \mathrm{ii}), 3.6 \widetilde{\mathfrak{U}}=\mathfrak{U}, \widetilde{P}=P(\widetilde{\mathfrak{F}}=\mathfrak{F}, \widetilde{Q}=Q)$.

СЛЕДСТВИЕ 5.2. Пусть оператор $M$ сильно $(L, p)$-секториален справа (слева). Тогда $P=\mathrm{s}-\lim U(\tau) \quad(Q=\mathrm{s}-\lim F(\tau))$ при $|\tau| \rightarrow 0$ в секторе $\Sigma_{\theta-\delta}, \delta>0$.

ДоказАТЕльство. Сужение полугруппы $\left\{U(\tau): \tau \in \Sigma_{\theta}\right\}$ на подпространство $\mathfrak{U}^{1}$ дает полугруппу класса $H(-\theta+\pi / 2, \theta-\pi / 2)$ [15; гл. Х, определение 10.6.1]. Осталось к ней применить теорему 10.6.6 [15; гл. Х].

СЛЕДСТВИЕ 5.3. Пусть оператор $M$ сильно $(L, p)$-секториален справа и слева. Тогда

i) $\forall u \in \mathfrak{U} L P u=Q L u$;

ii) $\forall u \in \operatorname{dom} M \quad P u \in \operatorname{dom} M, M P u=Q M u$.

ДоказАТЕЛьство. Возьмем произвольньй вектор $u \in \operatorname{dom} M$. С учетом замкнутости оператора $M$ и того,что существуют пределы

$$
\lim _{t \rightarrow 0+} U(t) u=P u, \quad \lim _{t \rightarrow 0+} M U(t) u=\lim _{t \rightarrow 0+} F(t) M u=Q M u
$$

имеем второе утверждение леммы. Утверждение і) доказьвается аналогично.

Обозначим $L_{1}=\left.L\right|_{\mathfrak{U}^{1}}, M_{1}=\left.M\right|_{\mathrm{dom} M_{1}}, \operatorname{dom} M_{1}=\operatorname{dom} M \cap \mathfrak{U}^{1}$.

СЛЕДСТВИЕ 5.4. Пусть оператор $M$ сильно $(L, p)$-секториален справа и слева. Тогда $L_{1} \in \mathscr{L}\left(\mathfrak{U}^{1} ; \mathfrak{F}^{1}\right), M_{0} \in \mathrm{Cl}\left(\mathfrak{U}^{0} ; \mathfrak{F}^{0}\right)-$ биекция, $M_{1} \in \mathrm{Cl}\left(\mathfrak{U}^{1} ; \mathfrak{F}^{1}\right)$.

ДокАЗАТЕЛЬство. С учетом предыдушего следствия достаточно заметить лишь, что биективность оператора $M_{0}: \operatorname{dom} M_{0} \rightarrow \mathfrak{U}^{0}$ вытекает из следствия 3.1,i). Плотная определенность операторов $M_{0}, M_{1}$ следует из того, что для $u \in \operatorname{dom} M$ имеем $(I-P) u, P u \in \operatorname{dom} M$.

Лемма 5.1. Пусть оператор $M$ сильно (L,p)-секториален справа и слева. Тогда существует оператор $L_{1}^{-1} \in \mathrm{Cl}\left(\mathfrak{F}^{1} ; \mathfrak{U}^{1}\right)$. 
ДокАЗАТЕЛЬСтво. Инъективность оператора $L_{1}$ следует из того факта, что $\operatorname{ker} L \subset \mathfrak{U}^{0}$. Далее, $\left(L_{\mu}^{L}(M)\right)^{p+1}=\left(G(\mu G-I)^{-1}\right)^{p+1}(I-Q)+\left(L_{\mu}^{L_{1}}\left(M_{1}\right)\right)^{p+1} Q$. Так как $G$ нильпотентен степени не больше $p$ в силу следствия $3.1, \mathrm{ii})$, то im $L_{(\mu, p)}^{L}(M)=$ $\operatorname{im} L_{(\mu, p)}^{L_{1}}\left(M_{1}\right), \mathfrak{F}^{1} \subset \overline{\operatorname{im} L_{1}}$. Но $\operatorname{im} L_{1} \subset \mathfrak{F}^{1}$, следовательно, $\operatorname{im} L_{1}$ плотен в $\mathfrak{F}^{1}$, а значит, $L_{1}^{-1}$ плотно определен.

ЗАмЕЧАнИЕ 5.2. Утверждения следствий 5.2-5.4 и леммы 5.1 останутся в силе, если заменить требование сильной $(L, p)$-секториальности оператора $M$ справа (слева) на требования $(L, p)$-секториальности оператора и полурефлексивности пространства $\mathfrak{U}(\mathfrak{F})$.

\section{§6. Существование обратного оператора}

Укажем условия существования оператора $L_{1}^{-1} \in \mathscr{L}\left(\mathfrak{F}^{1} ; \mathfrak{U}^{1}\right)$. Для этого определим семейство операторов $\left\{R(\tau) \in \mathscr{L}(\mathfrak{F} ; \mathfrak{U}): \tau \in \Sigma_{\theta}\right\}$,

$$
R(\tau)=\frac{1}{2 \pi i} \int_{\Gamma}(\mu L-M)^{-1} e^{\mu \tau} d \mu
$$

где $\Sigma_{\theta}=\{\tau \in \mathbb{C}:|\arg \tau|<\theta-\pi / 2\}$, контур $\Gamma$ удовлетворяет (2.2), а оператор $M$ $(L, p)$-секториален (интеграл сходится по лемме 1.6).

Лемма 6.1. Пусть оператор $M(L, p)$-секториален. Тогда семейство операторов $\left\{R(\tau): \tau \in \Sigma_{\theta}\right\}$ голоморфно в секторе $\Sigma_{\theta}$.

Лемма 6.2. Пусть оператор $M(L, p)$-секториален. Тогда

i) $\forall \tau \in \Sigma_{\theta} \quad R(\tau) L=U(\tau), L R(\tau)=F(\tau)$;

ii) $\forall \sigma, \tau \in \Sigma_{\theta} \quad R(\sigma+\tau)=U(\sigma) R(\tau)=R(\tau) F(\sigma)$.

Утверждение і) очевидно, ii) доказьвается так же, как полугрупповое свойство в теореме 2.1.

Лемма 6.3. Пусть оператор $M$ сильно $(L, p)$-секториален справа (слева). Тогда $R(\tau)=P R(\tau) \quad(R(\tau)=R(\tau) Q) \quad \forall \tau \in \Sigma_{\theta}$.

Для доказательства устремим в утверждении леммы 6.2 , ii) $\sigma$ к нулю справа.

Лемма 6.4. Пусть оператор $M$ сильно $(L, p)$-секториален справа (слева). Тогда $\overline{\bigcup_{t>0} \operatorname{im} R(t)}=\mathfrak{U}^{1}\left(\forall \tau \in \Sigma_{\theta} \quad \operatorname{ker} R(\tau)=\mathfrak{F}^{0}\right)$.

Доказательство. Очевидно, что $\forall t \in \mathbb{R}_{+} \operatorname{im} U(t) \subset \operatorname{im} R(t)$. В силу леммы 3.5 и теоремы 3.2 получаем, что $\mathfrak{U}^{1} \subset \overline{\bigcup_{t>0} \mathrm{im} R(t)}$. По лемме $6.3 \forall t \in \mathbb{R}_{+}$ $\operatorname{im} R(t) \subset \mathfrak{U}^{1}$. Из замкнутости подпространства $\mathfrak{U}^{1}$ получаем требуемое.

Пусть $\tau \in \Sigma_{\theta}, f \in \mathfrak{F}^{0}$. Тогда по лемме $6.3 R(\tau) f=R(\tau) Q f=0$. Поэтому $\mathfrak{F}^{0} \subset \operatorname{ker} R(\tau)$. Если же $R(\tau) f=0$, то $F(\tau) f=L R(\tau) f=0$ в силу леммы $\left.6.2, \mathrm{i}\right)$. Из теоремы 3.1 следует, что $\operatorname{ker} R(\tau) \subset \mathfrak{F}^{0}$.

Непрерьвньй оператор $L_{1}^{-1}$ нам необходим для того, чтобы решать неоднородное уравнение $L \dot{u}(t)=M u(t)+f(t)$ без дополнительного и весьма ограничительного требования $Q f(t) \in \mathrm{im} L_{1}$ (см. $\left.\S 8\right)$. Для получения этого оператора придется добавить еше одно условие на операторы $L, M$. Однако дополнительные требования на операторы в определениях 5.1 и 6.1 вписываются в рамки обобщения секториального оператора (см. по этому поводу замечание 6.3). 
ОПРЕДЕЛЕНИЕ 6.1. Оператор $M$ называется сильно $(L, p)$-секториальным, если он сильно $(L, p)$-секториален слева и эквинепрерьвно семейство операторов

$$
\begin{aligned}
\{(\lambda-a) & \prod_{k=0}^{p}\left(\mu_{k}-a\right) R_{(\mu, p)}^{L}(M)(\lambda L-M)^{-1}: \\
& \left.\lambda \in S_{a, \theta}^{L}(M), \mu=\left(\mu_{0}, \ldots, \mu_{p}\right) \in\left(S_{a, \theta}^{L}(M)\right)^{p+1}\right\} .
\end{aligned}
$$

ЗАмечание 6.1. Сильно $(L, p)$-секториальный оператор $M$ сильно $(L, p)$-секториален справа $\left(c_{1}(u)=r(M u)\right)$.

ЗАмечание 6.2 . В случае $L^{-1} \in \mathscr{L}(\mathfrak{F} ; \mathfrak{U})$ оператор $M$ сильно $(L, p)$-секториален, если секториален оператор $L^{-1} M$ (или $\left.M L^{-1}\right)$. В качестве плотного в пространстве $\mathfrak{F}$ линеала $\stackrel{\circ}{\mathfrak{F}}$ можно взять $L[\operatorname{dom} M]$.

Лемма 6.5. Пусть оператор $M$ сильно $(L, p)$-секториален. Тогда для любого $\delta \in(0, \pi / 2-\theta)$ семейство операторов $\left\{R(\tau): \tau \in \Sigma_{\theta-\delta}\right\}$ әкспоненциально ограничено.

ДокАЗАТЕЛЬСТВо аналогично доказательству экспоненциальной ограниченности полугруппы в теореме 2.1 .

Теорема 6.1. Пусть оператор $M$ сильно $(L, p)$-секториален. Тогда сущесmвует оператор $L_{1}^{-1} \in \mathscr{L}\left(\mathfrak{F}^{1} ; \mathfrak{U}^{1}\right)$.

ДокАЗАТЕЛЬСТво. Пусть $f \in \mathfrak{F}, s>t>0$. Тогда для любого $f \in \mathfrak{F}$ и для любой непрерывной на пространстве $\mathfrak{U}$ полунормы $q(\cdot)$

$$
q(R(s) f-R(t) f)=q(R(t)(F(s-t)-Q) f) \leqslant e^{a t} r((F(s-t)-Q) f)
$$

в силу лемм $6.2,6.3$ и 6.5 . Здесь $r(\cdot)$ - непрерывная на пространстве $\mathfrak{F}$ полунорма. Отсюда следует сушествование оператора $R(0)=\mathrm{s}^{-\lim _{t \rightarrow 0+}} R(t) \in \mathscr{L}(\mathfrak{F} ; \mathfrak{U})$, который непрерывен как предельный для эквинепрерьвного семейства операторов $\{R(t) \in \mathscr{L}(\mathfrak{F} ; \mathfrak{U}): t \in(0, \delta)\}$. Согласно лемме $6.2 R(0) L=P$ и $L R(0)=Q$. Сузив эти тождества на $\mathfrak{U}^{1}$ и $\mathfrak{F}^{1}$ соответственно, получим $R_{1}(0) L_{1}=I, L_{1} R_{1}(0)=I$, где $R_{1}(0)=\left.R(0)\right|_{\mathfrak{F}^{1}}$. Таким образом, $R_{1}(0)=L_{1}^{-1} \in \mathscr{L}\left(\mathfrak{F}^{1} ; \mathfrak{U}^{1}\right)$.

Как уже было замечено, сужение $\left\{U_{1}(\tau): \tau \in \Sigma_{\theta}\right\} \quad\left(\left\{F_{1}(\tau): \tau \in \Sigma_{\theta}\right\}\right)$ полугрупшы $\left\{U(\tau): \tau \in \Sigma_{\theta}\right\} \quad\left(\left\{F(\tau): \tau \in \Sigma_{\theta}\right\}\right)$ на подпространство $\mathfrak{U}^{1}\left(\mathfrak{F}^{1}\right)$ является полугруппой класса $H(-\theta+\pi / 2, \theta-\pi / 2)$ [15; гл. Х, определение 10.6.1].

Обозначим $S_{1}=L_{1}^{-1} M_{1} \in \mathrm{Cl}\left(\mathfrak{U}^{1}\right), \operatorname{dom} S_{1}=\operatorname{dom} M_{1} ; T_{1}=M_{1} L_{1}^{-1} \in \mathrm{Cl}\left(\mathfrak{F}^{1}\right)$, $\operatorname{dom} T_{1}=L_{1}\left[\operatorname{dom} M_{1}\right]$. Ясно, что при условии сильной $(L, p)$-секториальности оператора $M$ множество $L_{1}\left[\operatorname{dom} M_{1}\right]$ плотно в $\mathfrak{F}^{1}$ в силу теоремы 6.1 .

Теорема 6.2. Пусть оператор $M$ сильно $(L, p)$-секториален. Тогда инфинитезимальным генератором полугруппы $\left\{U_{1}(\tau): \tau \in \Sigma_{\theta}\right\} \quad\left(\left\{F_{1}(\tau): \tau \in \Sigma_{\theta}\right\}\right)$ является оператор $S_{1}\left(T_{1}\right)$. 
ДоКАЗАТЕЛЬСТво. Из интегрального представления полугрупп (2.1), теоремы 6.1 и тождеств $R_{\mu}^{L_{1}}\left(M_{1}\right)=R_{\mu}\left(S_{1}\right), L_{\mu}^{L_{1}}\left(M_{1}\right)=R_{\mu}\left(T_{1}\right)$ следует, что сужения полугрупп могут быть представлены в виде

$$
U_{1}(\tau)=\frac{1}{2 \pi i} \int_{\Gamma} R_{\mu}\left(S_{1}\right) e^{\mu \tau} d \mu, \quad F_{1}(\tau)=\frac{1}{2 \pi i} \int_{\Gamma} R_{\mu}\left(T_{1}\right) e^{\mu \tau} d \mu
$$

где $R_{\mu}(A)=(\mu I-A)^{-1}$. Осталось сослаться на теорему Иосиды о генераторах голоморфных полугрупп.

СлеДСТВИЕ 6.1. Пусть оператор $M$ сильно $(L, p)$-секториален. Тогда операторы $S_{1}$ и $T_{1}$ секториальны.

ЗамечаниЕ 6.3. Если $\mathfrak{U}=\mathfrak{F}$, то согласно следствию 6.1 оператор $M$ сильно $(I, p)$-секториален при некотором $p \in\{0\} \cup \mathbb{N}$ тогда и только тогда, когда он секториален.

ЗАмЕЧАниЕ 6.4. Если условие сильной $(L, p)$-секториальности заменить на условие сильной $(L, p)$-секториальности справа и слева и взять при этом $\operatorname{dom} S_{1}=$ $\operatorname{im}\left(\mu L_{1}-M_{1}\right)^{-1} L_{1}, \operatorname{dom} T_{1}=\operatorname{im} L_{1}\left(\mu L_{1}-M_{1}\right)^{-1}$, то утверждения теоремы 6.2 , следствия 6.1 и замечания 6.3 также будут справедливы. При этом в случае полурефилексивных пространств $\mathfrak{U}$ и $\mathfrak{F}$ можно ограничиться требованием $(L, p)$-секториальности оператора $M$ (см. замечание 5.2).

\section{§7. Генераторы вырожденных сильно голоморфных полугрупп}

Пусть $\mathfrak{U}, \mathfrak{F}$ - секвенциально полные локально выпуклые линейные топологические пространства и вьполняются следующие условия.

УСловие Н1. Существует пара

$$
(\{U(\tau) \in \mathscr{L}(\mathfrak{U}): \tau \in \Sigma \cup\{0\}\},\{F(\tau) \in \mathscr{L}(\mathfrak{F}): \tau \in \Sigma \cup\{0\}\})
$$

сильно голоморфных в секторе $\Sigma=\{\tau \in \mathbb{C}:|\arg \tau|<\varepsilon\}, \varepsilon \in(0, \pi / 2)$, сильно непрерывных на $\overline{\mathbb{R}}_{+}$и әкспоненциально ограниченных полугрупп операторов.

Положим $P=U(0), Q=F(0), \mathfrak{U}^{0}=\operatorname{ker} P, \mathfrak{U}^{1}=\operatorname{im} P, \mathfrak{F}^{0}=\operatorname{ker} Q, \mathfrak{F}^{1}=\operatorname{im} Q$. Имеем $\mathfrak{U}=\mathfrak{U}^{0} \oplus \mathfrak{U}^{1}, \mathfrak{F}=\mathfrak{F}^{0} \oplus \mathfrak{F}^{1}$.

Через $\left\{U_{1}(\tau) \in \mathscr{L}\left(\mathfrak{U}^{1}\right): \tau \in \Sigma \cup\{0\}\right\}$ и $\left\{F_{1}(\tau) \in \mathscr{L}\left(\mathfrak{F}^{1}\right): \tau \in \Sigma \cup\{0\}\right\}$ обозначим сужения соответствуюших полугрупп на подпространства $\mathfrak{U}^{1}$ и $\mathfrak{F}^{1}$. Сужения являются невырожденными голоморфными полугруппами, и по теореме Иосиды они имеют инфинитезимальные генераторы $S_{1} \in \mathrm{Cl}\left(\mathfrak{U}^{1}\right)$ и $T_{1} \in \mathrm{Cl}\left(\mathfrak{F}^{1}\right)$ соответственно, являюшиеся секториальными операторами.

УСловиЕ Н2. Существует линейный гомеоморфизм $L_{1}: \mathfrak{U}^{1} \rightarrow \mathfrak{F}^{1}$ такой, что $\operatorname{dom} T_{1}=L_{1}\left[\operatorname{dom} S_{1}\right]$ и для всех $u \in \operatorname{dom} S_{1} L_{1} S_{1} u=T_{1} L_{1} u$.

УСловИе Н3. Существует инбективный оператор $N_{0} \in \mathscr{L}\left(\mathfrak{F}^{0} ; \mathfrak{U}^{0}\right)$ с плотныцм в $\mathfrak{U}^{0}$ образом.

Отсюда следует существование биективного оператора $M_{0}=N_{0}^{-1} \in \mathrm{Cl}\left(\mathfrak{U}^{0} ; \mathfrak{F}^{0}\right)$ с областью определения $\operatorname{dom} M_{0}=\operatorname{im} N_{0}$. 
УСловие Н4. Существует такой оператор $L_{0} \in \mathscr{L}\left(\mathfrak{U}^{0} ; \mathfrak{F}^{0}\right)$, что оператор $H=M_{0}^{-1} L_{0}$ нильпотентен степени не больше $p \in\{0\} \cup \mathbb{N}$.

УСловие Н5. Справедливо следующее:

$$
\begin{gathered}
L=L_{0}(I-P)+L_{1} P ; \quad M=M_{0}(I-P)+L_{1} S_{1} P, \\
\operatorname{dom} M=\operatorname{dom} M_{0} \dot{+} \operatorname{dom} S_{1} .
\end{gathered}
$$

Tеорема 7.1. Оператор $M$ сильно $(L, p)$-секториален тогда и только тогда, когда выполнены условия $\mathrm{H} 1-\mathrm{H} 5$.

ДокАЗАТЕЛЬСтво. Необходимость условий показана в предыдущих параграфах. Достаточность следует из секториальности операторов $S_{1}, T_{1}$, нильпотентности оператора $H$ и тождеств

$$
\begin{gathered}
(\mu L-M)^{-1}=-\sum_{k=0}^{p} \mu^{k} H^{k} M_{0}^{-1}(I-Q)+\left(\mu I-S_{1}\right)^{-1} L_{1}^{-1} Q \\
R_{(\nu, p)}^{L}(M)=\prod_{k=0}^{p} R_{\nu_{k}}\left(S_{1}\right) P, \quad L_{(\nu, p)}^{L}(M)=\prod_{k=0}^{p} R_{\nu_{k}}\left(T_{1}\right) Q \\
M(\lambda L-M)^{-1} L_{(\nu, p)}^{L}(M) f=R_{\lambda}\left(T_{1}\right) \prod_{k=0}^{p} R_{\nu_{k}}\left(T_{1}\right) T_{1} Q f \\
R_{(\nu, p)}^{L}(M)(\lambda L-M)^{-1}=R_{\lambda}\left(S_{1}\right) \prod_{k=0}^{p} R_{\nu_{k}}\left(S_{1}\right) L_{1}^{-1} P u
\end{gathered}
$$

где $\mu, \lambda, \nu_{k} \in S_{a, \theta}^{L}(M), k=\overline{0, p}, f \in \stackrel{\circ}{\mathfrak{F}}=\mathfrak{F}^{0} \dot{+} \operatorname{dom} T_{1}$.

СлЕДСТВИЕ 7.1. Из сильной $(L, p)$-секториальности оператора $M$ следует его сильная $(L, p+q)$-секториальность при $q \in \mathbb{N}$.

ДокАЗАТЕЛЬСТво.Действительно, параметр $p$ фигурирует лишњ в условии $\mathrm{H} 4$ и может быть увеличен произвольным образом.

Введем в рассмотрение еще одно

УСловие Н2' . Существует инбективныий оператор $L_{1} \in \mathscr{L}\left(\mathfrak{U}^{1} ; \mathfrak{F}^{1}\right)$ с плотныцм в $\mathfrak{U}^{1}$ образом, при этом $L_{1}\left[\operatorname{dom} S_{1}\right] \subset \operatorname{dom} T_{1} \subset \operatorname{im} L_{1}$, и для всех $u \in \operatorname{dom} S_{1} \quad L_{1} S_{1} u=T_{1} L_{1} u$.

Теорема 7.2. Оператор $M$ (L,p)-секториален справа и слева тогда и только тогда, когда выполненьг условия $\mathrm{H} 1, \mathrm{H} 2^{\prime}, \mathrm{H} 3-\mathrm{H} 5$.

Для доказательства необходимости условия $\mathrm{H} 2^{\prime}$ надо положить $\operatorname{dom} S_{1}=$ $\operatorname{im}\left(\mu L_{1}-M_{1}\right)^{-1} L_{1}, \operatorname{dom} T_{1}=\operatorname{im} L_{1}\left(\mu L_{1}-M_{1}\right)^{-1}$ и учесть замечание 6.4.

ЗАмечАнИЕ 7.1. Каждая из теорем 7.1, 7.2 является обобщением теоремы Иосиды об инфинитезимальных генераторах голоморфыш полугрупп операторов, поскольку при $\mathfrak{U}=\mathfrak{F}, L=I$ условия $\mathrm{H} 2-\mathrm{H} 5, \mathrm{H} 2^{\prime}$ становятся тривиальными, а сильная $(I, p)$-секториальность в точности означает секториальность оператора $M=S_{1}=T_{1}$ согласно замечаниям 6.3 и 6.4 .

Из замечаний 5.2 и 6.4 следует 
ТЕОРема 7.3. Если пространства $\mathfrak{U}$ и $\mathfrak{F}$ полурефлексивны, то оператор $M$ $(L, p)$-секториален в том и только том случае, если выполнены условия $\mathrm{H} 1$, $\mathrm{H} 2^{\prime}, \mathrm{H} 3-\mathrm{H} 5$.

СЛЕДСТВИЕ 7.2. Если пространства $\mathfrak{U}$ u $\mathfrak{F}$ полурефлексивны, то оператор $M(L, p)$-секториален в том и только том случае, если он сильно $(L, p)$-секториален справа и слева.

СЛЕДСТВИЕ 7.3. і) Из сильной $(L, p)$-секториальности оператора $M$ справа и слева следует его сильная $(L, p+q)$-секториальность справа и слева при любом $q \in \mathbb{N}$;

ii) если пространства $\mathfrak{U}$ и $\mathfrak{F}$ полурефлексивнь, то из $(L, p)$-секториальности оператора $M$ следует его $(L, p+q)$-секториальность при любом $q \in \mathbb{N}$.

Положим $\mathbb{C}_{a,+}=\{\mu \in \mathbb{C}: \operatorname{Re} \mu>a\}$.

ТЕОРема 7.4. Пусть пространства $\mathfrak{U}$ u $\mathfrak{F}$ полурефлексивны. Тогда оператор $M(L, p)$-секториален в том и только том случае, если $\mathbb{C}_{a,+} \subset \rho^{L}(M)$ и найдется константа $C>0$ такая, что семейства операторов

$$
\begin{aligned}
& \left\{\left(C^{p+1} R_{(\mu, p)}^{L}(M) \prod_{k=0}^{p}\left(\mu_{k}-a\right)\right)^{n}: \mu=\left(\mu_{0}, \ldots, \mu_{p}\right) \in \mathbb{C}_{a,+}^{p+1}, n \in \mathbb{N}\right\}, \\
& \left\{\left(C^{p+1} L_{(\mu, p)}^{L}(M) \prod_{k=0}^{p}\left(\mu_{k}-a\right)\right)^{n}: \mu=\left(\mu_{0}, \ldots, \mu_{p}\right) \in \mathbb{C}_{a,+}^{p+1}, n \in \mathbb{N}\right\}, \\
& \left\{\prod_{k=0}^{p}\left(C\left(\mu_{k}-a\right) R_{\mu_{k}}^{L}(M)\right)^{l_{k}+1}:\left(\mu_{0}, \ldots, \mu_{p}\right) \in \mathbb{C}_{a,+}^{p+1}, l_{0}, \ldots, l_{p} \in \overline{0, p}\right\}, \\
& \left\{\prod_{k=0}^{p}\left(C\left(\mu_{k}-a\right) L_{\mu_{k}}^{L}(M)\right)^{l_{k}+1}:\left(\mu_{0}, \ldots, \mu_{p}\right) \in \mathbb{C}_{a,+}^{p+1}, l_{0}, \ldots, l_{p} \in \overline{0, p}\right\}
\end{aligned}
$$

эквинепрерьвны.

ДоказАТЕльство. Возьмем $\lambda \in \mathbb{C}, \mu \in \mathbb{C}_{a,+} \cdot$ Тогда

$$
q\left(\left((\lambda-\mu) L_{\mu}^{L}(M)\right)^{k(p+1)} f\right) \leqslant \frac{|\lambda-\mu|^{k(p+1)}}{(C|\mu-a|)^{k(p+1)}} r(f)
$$

при всех $f \in \mathfrak{F}, k \in \mathbb{N}$. Отсюда имеем при $|\lambda-\mu|<C|\mu-a|$

$$
(\lambda L-M)^{-1} f=(\mu L-M)^{-1} \sum_{n=0}^{\infty}(\mu-\lambda)^{n}\left(L_{\mu}^{L}(M)\right)^{n} f .
$$


Действительно, из (1.1) и (7.2) следует

$$
\begin{aligned}
(\lambda L-M)^{-1} & =(\mu L-M)^{-1}\left(I-(\mu-\lambda) L_{\mu}^{L}(M)\right)^{-1} \\
q\left((\lambda L-M)^{-1} f\right) & \leqslant \sum_{n=0}^{\infty}|\lambda-\mu|^{n} q\left((\mu L-M)^{-1}\left(L_{\mu}^{L}(M)\right)^{n} f\right) \\
& =\sum_{l=0}^{p} \sum_{k=0}^{\infty}|\lambda-\mu|^{k(p+1)+l} q\left((\mu L-M)^{-1}\left(L_{\mu}^{L}(M)\right)^{k(p+1)+l} f\right) \\
& \leqslant \sum_{l=0}^{p} \sum_{k=0}^{\infty}|\lambda-\mu|^{k(p+1)+l} q_{l}\left(\left(L_{\mu}^{L}(M)\right)^{k(p+1)} f\right) \\
& <\sum_{l=0}^{p} C^{l}|\mu-a|^{l} \sum_{k=0}^{\infty}\left|\frac{\lambda-\mu}{C(\mu-a)}\right|^{k(p+1)} r_{l}(f) \\
& =\left(1-\frac{|\lambda-\mu|^{p+1}}{(C|\mu-a|)^{p+1}}\right)^{-1} \sum_{l=0}^{p} C^{l}|\mu-a|^{l} r_{l}(f) .
\end{aligned}
$$

Здесь использована непрерьвность операторов $(\mu L-M)^{-1}\left(L_{\mu}^{L}(M)\right)^{l}, l=\overline{0, p}$, означаюшая, что для всякой непрерывной в $\mathfrak{U}$ полунормы $q(\cdot)$ сушествует непрерывная в $\mathfrak{F}$ полунорма $q_{l}(\cdot)$ такая, что для всех $g \in \mathfrak{F}$ вьполняется

$$
q\left((\mu L-M)^{-1}\left(L_{\mu}^{L}(M)\right)^{l} g\right) \leqslant q_{l}(g) .
$$

Покажем, что точки $\lambda=a-\delta C|y|+i y, y \in \mathbb{R} \backslash\{0\}, \delta \in(0,1)$, лежат в $\rho^{L}(M)$. Выберем $\mu=a+\varepsilon+i y, \varepsilon(y) \in(0,(1-\delta) C|y|)$. Тогда

$$
|\lambda-\mu|=\varepsilon+\delta C|y|<C|y|<C|\mu-a| .
$$

Поэтому любая пара лучей вида $\{\mu \in \mathbb{C}:|\arg (\mu-a)|=\pi / 2+\operatorname{arctg}(\delta C), \mu \neq a\}$ лежит в $\rho^{L}(M)$. Отсюда

$$
S_{a, \theta}^{L}(M)=\left\{\mu \in \mathbb{C}:|\arg (\mu-a)|<\theta=\frac{\pi}{2}+\operatorname{arctg} C\right\} \subset \rho^{L}(M) .
$$

Далее, при $\left|\lambda_{p}-\mu_{p}\right| \leqslant \delta C\left|\mu_{p}-a\right|, \delta \in(0,1), u \in \mathfrak{U}$ из (7.4) следует

$$
q\left(R_{\lambda_{p}}^{L}(M) u\right) \leqslant \sum_{l=0}^{p} \frac{\left|\lambda_{p}-\mu_{p}\right|^{l}}{1-\delta^{p+1}} r\left(\left(R_{\mu_{p}}^{L}(M)\right)^{l+1} u\right) .
$$

Положим здесь $u=R_{(\lambda, p-1)}^{L}(M) v$. Тогда при $C_{1}=\left(1-\delta^{p+1}\right)^{-1}$

$$
\begin{aligned}
q\left(R_{(\lambda, p)}^{L}(M) v\right) & \leqslant C_{1}^{p+1} \sum_{l_{0}=0}^{p}\left|\lambda_{0}-\mu_{0}\right|^{l_{0}} \cdots \sum_{l_{p}=0}^{p}\left|\lambda_{p}-\mu_{p}\right|^{l_{p}} r_{1}\left(\prod_{k=0}^{p}\left(R_{\mu_{k}}^{L}(M)\right)^{l_{k}+1} v\right) \\
& \leqslant \frac{C_{1}^{p+1}}{C^{p+1} \prod_{k=0}^{p}\left|\mu_{k}-a\right|} \sum_{l_{0}=0}^{p} \cdots \sum_{l_{p}=0}^{p} \delta^{\sum_{m=0}^{p} l_{m}} r_{2}(v) \\
& <\left(\frac{C_{1}(p+1)(1+\delta C)}{C}\right)^{p+1} \frac{r_{2}(v)}{\prod_{k=0}^{p}\left(\lambda_{k}-a\right)} .
\end{aligned}
$$


При этом мы использовали условие (7.3) и неравенство $\left|\lambda_{k}-a\right| \leqslant\left|\lambda_{k}-\mu_{k}\right|+$ $\left|\mu_{k}-a\right|<(1+\delta C)\left|\mu_{k}-a\right|, k=\overline{0, p}$. Возьмем непрерьвную полунорму

$$
r(\cdot)=\left(\frac{C_{1}(p+1)(1+\delta C)}{C}\right)^{p+1} r_{2}(\cdot)
$$

и получим, что $q\left(R_{(\lambda, p)}^{L}(M) \prod_{k=0}^{p}\left(\lambda_{k}-a\right) v\right) \leqslant r(v)$ для $\lambda_{k} \in S_{a, \theta}^{L}(M), k=\overline{0, p}$.

Итак, даже не используя полурефлексивность, мы получили, что оператор $M$ $(L, p)$-секториален с константой $\theta=\pi / 2+\operatorname{arctg}(\delta C)$ при любом $\delta \in(0,1)$.

Необходимость условий (7.2), (7.3) следует из тождеств (7.1) (с возможной заменой числа $p$ на большее) и секториальности операторов $S_{1}$ и $T_{1}$ (см. замечание 6.4). Действительно, из их секториальности в свою очередь следует эквинепрерывность семейств

$$
\left\{\left(C(\mu-a) R_{\mu}\left(S_{1}\right)\right)^{n}: \mu \in \mathbb{C}_{a,+}, n \in \mathbb{N}\right\}, \quad\left\{\left(C(\mu-a) R_{\mu}\left(T_{1}\right)\right)^{n}: \mu \in \mathbb{C}_{a,+}, n \in \mathbb{N}\right\}
$$

при некотором $C>0[1 ;$ гл. IX, $\S 10]$.

ЗАмЕчАниЕ 7.2. Можно показать, что во всех утверждениях данного параграфа условие полурефлексивности пространств $\mathfrak{U}$ и $\mathfrak{F}$ можно заменить на условия $\mathfrak{U}=\mathfrak{U}^{0} \oplus \mathfrak{U}^{1}, \mathfrak{F}=\mathfrak{F}^{0} \oplus \mathfrak{F}^{1}$.

\section{§8. Неоднородная задача Коши}

Рассмотрим ослабленную задачу Коши $u(0)=u_{0}$ для неоднородного линейного уравнения соболевского типа

$$
L \dot{u}(t)=M u(t)+f(t)
$$

с сильно $(L, p)$-секториальным оператором $M$.

Пусть $\mathfrak{V}$ - локально выпуклое пространство, вектор-функцию $g: \mathbb{R}_{+} \rightarrow \mathfrak{V}$ будем называть локально гёльдеровой, если для любой непрерывной в пространстве $\mathfrak{V}$ полунормы $q(\cdot)$ и для любого $t \in \mathbb{R}_{+}$найдутся окрестность $\mathscr{O}_{t}$ точки $t$ и константы $C_{q, t}, \alpha_{q, t}>0$ такие, что для любого $s \in \mathscr{O}_{t} q(g(t)-g(s)) \leqslant C_{q, t}|t-s|^{\alpha_{q, t}}$.

ЛЕмма 8.1. Пусть $\mathfrak{V}$ - секвенциально полное локально выпуклое пространство, оператор А секториален, вектор-функиия $g: \mathbb{R}_{+} \rightarrow \mathfrak{V}$ локально гёльдерова и при некотором $\rho(q) \int_{0}^{\rho(q)} q(g(t)) d t<\infty$. Тогда для любого начального значения $v_{0} \in \mathfrak{V}$ существует единственное решение ослабленной задачи Коши $v(0)=v_{0}$ для уравнения $\dot{v}(t)=A v(t)+g(t), t \in \mathbb{R}_{+}$, имеющее вид

$$
v(t)=V(t) v_{0}+\int_{0}^{t} V(t-s) g(s) d s \in C^{1}\left(\mathbb{R}_{+}, \mathfrak{V}\right) \cap C\left(\overline{\mathbb{R}}_{+}, \mathfrak{V}\right) .
$$

Аналогичное утверждение в случае банахова пространства $\mathfrak{V}$ доказано в $[2 ;$ гл. 3 , $\S 3.2]$. Доказательство леммы 8.1 отличается от приведенных в [2] рассуждений только заменой нормы на произвольную непрерьвную полунорму. Заметим, что, например, в [16; гл. $5, \S 4]$ такое же утверждение для банахова пространства доказано при более сильном условии гёльдеровости вектор-функции $g$. Однако из приведенного в [16] доказательства видно, что это условие можно ослабить. 
Tеорема 8.1. Пусть оператор $M$ сильно $(L, p)$-секториален, вектор-функиия $f$ такова, что $f^{0}=(I-Q) f \in C^{p+1}\left(\mathbb{R}_{+}, \mathfrak{F}\right) \cap C^{p}\left(\overline{\mathbb{R}}_{+}, \mathfrak{F}\right)$, а функиия $g=L_{1}^{-1} Q f: \mathbb{R}_{+} \rightarrow \mathfrak{U}^{1}$ удовлетворяет условиям леммы 8.1. Тогда для любого начального значения

$$
u_{0} \in \mathfrak{P}_{f}=\left\{u \in \mathfrak{U}:(I-P) u=-\sum_{k=0}^{p} H^{k} M_{0}^{-1} f^{0(k)}(0)\right\}
$$

существует единственное решение $и \in C^{1}\left(\mathbb{R}_{+}, \mathfrak{U}\right) \cap C\left(\overline{\mathbb{R}}_{+}, \mathfrak{U}\right)$ ослабленной задачи Коши и $(0)=u_{0}$ для уравнения (8.1), причем

$$
u(t)=U(t) u_{0}+\int_{0}^{t} U(t-s) L_{1}^{-1} Q f(s) d s-\sum_{k=0}^{p} H^{k} M_{0}^{-1} f^{0(k)}(t) .
$$

ДокАЗАТЕЛЬСТво. Подействуем на (8.1) оператором $L_{1}^{-1} Q \in \mathscr{L}\left(\mathfrak{F} ; \mathfrak{U}^{1}\right)$ и получим уравнение

$$
\dot{u}(t)=S_{1} u(t)+L_{1}^{-1} Q f(t)
$$

на пространстве $\mathfrak{U}^{1}$ с секториальньм оператором $S_{1}=L_{1}^{-1} M_{1}$ в силу следствия 6.1. Поэтому согласно лемме 8.1 для любого $u_{0}^{1} \in \mathfrak{U}^{1}$ сушествует единственное ослабленное решение

$u(t)=U_{1}(t) P u_{0}+\int_{0}^{t} U_{1}(t-s) L_{1}^{-1} Q f(s) d s=U(t) u_{0}+\int_{0}^{t} U(t-s) L_{1}^{-1} Q f(s) d s$

задачи $u(0)=u_{0}^{1}=P u_{0}$ для уравнения (8.2).

Подействовав на уравнение (8.1) непрерьвньм оператором $M_{0}^{-1}(I-Q)$, получим уравнение $H \dot{u}(t)=u(t)+M_{0}^{-1} f^{0}(t)$ на пространстве $\mathfrak{U}^{0}$. Поочередно дифференцируя его и действуя оператором $H$ на обе части уравнения и используя нильпотентность оператора $H$, получим $u(t)=-\sum_{k=0}^{p} H^{k} M_{0}^{-1} f^{0(k)}(t)$. Для того чтобы это решение удовлетворяло условию $u(0)=(I-P) u_{0}=u_{0}^{0}$, необходимо и достаточно, чтобы $u_{0}^{0} \in \mathfrak{U}^{0} \cap \mathfrak{P}_{f}$.

\section{§9. Приложение к уравнениям в частных производных}

Рассмотрим банахово пространство $\mathfrak{V}$ и некоторый оператор $A \in \mathrm{Cl}(\mathfrak{V})$. Обозначим $\operatorname{dom} A^{\infty}=\bigcap_{k=1}^{\infty} \operatorname{dom} A^{k}$. Наделив это множество топологией, определяемой системой полунорм $q_{k}(u)=\sum_{l=0}^{k}\left\|A^{l} u\right\|_{\mathfrak{V}}, k \in \mathbb{N}_{0}=\{0\} \cup \mathbb{N}$, получим пространство Фреше, которое обозначим через $\mathfrak{D}_{A}$. Зафиксируем положительное число $\tau$. Пусть $\stackrel{\circ}{\mathfrak{E}}_{A}(\tau)=\left\{u \in \operatorname{dom} A^{\infty}: \varlimsup_{\overline{\lim }} \rightarrow \infty\left\|A^{k} u\right\|_{\mathfrak{V}}^{1 / k} \leqslant \tau\right\}$ - так называемое множество элементов $A$-экспоненциального типа, не превосходящего $\tau$. Возьмем наибольшее замкнутое в топологии $\mathfrak{D}_{A}$ подпространство, содержашееся в $\stackrel{\circ}{\mathfrak{E}}_{A}(\tau)$, и обозначим его через $\mathfrak{E}_{\tau}$. Множество $\mathfrak{E}_{\tau}$, наделенное топологией, определяемой полунормами $q_{k}(\cdot)$, также является пространством Фреше. Обозначим $A_{\tau}=\left.A\right|_{\mathfrak{E}_{\tau}}$. В работе $[17 ;$ гл. $7, \S 3]$ показано, что $\sigma\left(A_{\tau}\right) \subset \sigma(A) \cap B_{\tau}(0)$ и

$$
\exists C>0 \quad \forall \beta \in \mathbb{N}_{0} \exists \beta_{0} \in \mathbb{N}_{0} \quad \forall k \in \mathbb{N}, \quad \forall u \in \mathfrak{E}_{\tau} \quad q_{\beta}\left(A^{k} u\right) \leqslant C^{k} q_{\beta_{0}}(u) .
$$


Пусть $L=P(A)=\sum_{k=0}^{\infty} c_{k} A^{k}, M=Q(A)=\sum_{k=0}^{\infty} d_{k} A^{k}$, где $P(\lambda), Q(\lambda)-$ целые функции. Из сказанного выше сразу следует, что $L, M \in \mathscr{L}\left(\mathfrak{E}_{\tau}\right)$.

Рассмотрим индуктивную шкалу локально вьпуклых линейных топологических пространств $\left\{\mathfrak{E}_{\tau}: \tau \in \mathbb{N}\right\}$ и ее индуктивный предел $\mathfrak{E}_{\infty}$, который является внутренним и даже строгим [18; гл. I, дополнение]. Пространство $\mathfrak{E}_{\infty}=$ $\bigcup_{\tau \in \mathbb{N}} \mathfrak{E}_{\tau} \subset \mathfrak{D}_{A}$ имеет отделимую локально вьпуклую топологию, определяемую полунормами $q_{k, \tau}(u)=\sum_{l=0}^{k}\left\|A_{\tau}^{l} u\right\|_{\mathfrak{V}}, k \in \mathbb{N}_{0}, \tau \in \mathbb{N}$. Из предложения 4.1 [18; гл. I, дополнение] сразу следует

Лемма 9.1. Onераторь $P(A), Q(A) \in \mathscr{L}\left(\mathfrak{E}_{\infty}\right)$.

Обозначим $\mathscr{P}_{0}=\{\lambda \in \mathbb{R}: P(\lambda)=0\}$.

Теорема 9.1. Пусть $\mathfrak{V}$ - гильбертово пространство, $A$ - самосопряженный оператор, $\mathfrak{U}=\mathfrak{F}=\mathfrak{E}_{\infty}$, целье функции $P(\lambda), Q(\lambda)$ не имеют общих нулей на множестве $\sigma(A)$. Кроме того, пусть выполняются следующие условия:

$$
\begin{gathered}
\operatorname{dist}\left(\sigma(A) \backslash \mathscr{P}_{0}, \mathscr{P}_{0}\right)>0, \\
\exists b \in \mathbb{R}, \quad \exists \psi \in\left(0, \frac{\pi}{2}\right) \\
\frac{Q}{P}\left[\sigma(A) \backslash \mathscr{P}_{0}\right] \subset\left\{\mu \in \mathbb{C}:|\arg (\mu-b)| \in\left[\frac{\pi}{2}+\psi, \pi\right]\right\} .
\end{gathered}
$$

Тогда оператор $Q(A)$ сильно $(P(A), 0)$-секториален.

ДокАЗАТЕЛЬСтво. Возьмем в определении $(L, 0)$-секториального оператора $a=b, \theta=\pi / 2+\psi / 2$, при этом получим неравенство

$$
\left|\mu-\frac{Q(\lambda)}{P(\lambda)}\right| \geqslant|\mu-a| \sin \frac{\psi}{2} \quad \forall \lambda \in \mathbb{R} \backslash \mathscr{P}_{0}, \quad \forall \mu \in S_{a, \theta}^{L}(M) .
$$

Через $\left\{E_{\lambda}: \lambda \in \mathbb{R}\right\}$ обозначим спектральное семейство самосопряженного оператора $A$, а через $\langle\cdot, \cdot\rangle$ - скалярное произведение в пространстве $\mathfrak{V}$. Для $\beta \in \mathbb{N}_{0}$, $\tau \in \mathbb{N}, \mu \in S_{a, \theta}^{L}(M)$ имеем

$$
\begin{aligned}
& q_{\beta, \tau}\left(R_{\mu}^{L}(M) u\right)=\sum_{\gamma=0}^{\beta}\left\|\int_{\sigma\left(A_{\tau}\right) \backslash \mathscr{P}_{0}} \frac{\lambda^{\gamma} d E_{\lambda} u}{\mu-Q(\lambda) / P(\lambda)}\right\|_{\mathfrak{V}} \\
& \quad \leqslant \frac{\sin ^{-1}(\psi / 2)}{|\mu-a|} \sum_{\gamma=0}^{\beta} \sqrt{\int_{\sigma\left(A_{\tau}\right) \backslash \mathscr{P}_{0}} \lambda^{2 \gamma} d\left\langle E_{\lambda} u, u\right\rangle} \leqslant \frac{\sin ^{-1}(\psi / 2)}{|\mu-a|} q_{\beta, \tau}(u) .
\end{aligned}
$$

Далее, заметим, что в силу условий теоремы и непрерьвности функции $P(\lambda)$

$$
\exists c>0 \quad \forall \lambda \in \sigma(A) \backslash \mathscr{P}_{0} \quad|P(\lambda)| \geqslant c .
$$


Тогда для $f \in \mathfrak{E}_{\infty}$

$$
\begin{aligned}
q_{\beta, \tau}( & \left.M(\nu L-M)^{-1} L_{\mu}^{L}(M) f\right) \\
= & \sum_{\gamma=0}^{\beta}\left\|\int_{\sigma\left(A_{\tau}\right) \backslash \mathscr{P}_{0}} \frac{\lambda^{\gamma} Q(\lambda) d E_{\lambda} f}{P(\lambda)(\mu-Q(\lambda) / P(\lambda))(\nu-Q(\lambda) / P(\lambda))}\right\|_{\mathfrak{V}} \\
& \leqslant \frac{c^{-1} \sin ^{-2}(\psi / 2)}{|\mu-a||\nu-a|} \sum_{\gamma=0}^{\beta} \sqrt{\int_{\sigma\left(A_{\tau}\right)} \lambda^{2 \gamma}|Q(\lambda)|^{2} d\left\langle E_{\lambda} f, f\right\rangle} \\
& =\frac{c^{-1} \sin ^{-2}(\psi / 2)}{|\mu-a||\nu-a|} q_{\beta, \tau}(M f) .
\end{aligned}
$$

Таким образом, оператор $M$ сильно $(L, 0)$-секториален слева с константой $c_{2}(f)=$ $c^{-1} \sin ^{-2}(\psi / 2) \cdot q_{\beta, \tau}(M f)$ (см. определение 5.1). И, наконец,

$$
\begin{aligned}
q_{\beta, \tau} & \left(R_{\mu}^{L}(M)(\nu L-M)^{-1} f\right) \\
\quad= & \sum_{\gamma=0}^{\beta}\left\|\int_{\sigma\left(A_{\tau}\right) \backslash \mathscr{P}_{0}} \frac{\lambda^{\gamma} d E_{\lambda} f}{P(\lambda)(\mu-Q(\lambda) / P(\lambda))(\nu-Q(\lambda) / P(\lambda))}\right\|_{\mathfrak{V}} \\
\quad \leqslant & \frac{c^{-1} \sin ^{-2}(\psi / 2)}{|\mu-a||\nu-a|} \sum_{\gamma=0}^{\beta} \sqrt{\int_{\sigma\left(A_{\tau}\right)} \lambda^{2 \gamma} d\left\langle E_{\lambda} f, f\right\rangle}=\frac{c^{-1} \sin ^{-2}(\psi / 2)}{|\mu-a||\nu-a|} q_{\beta, \tau}(f) .
\end{aligned}
$$

ЗАмечание 9.1. Условие (9.1) означает, что множества $\sigma(A)$ и $\mathscr{P}_{0}$ пересекаются только в точках, являюшихся изолированными собственными значениями оператора $A$.

Таким образом, в условиях теоремы 9.1 в пространстве $\mathfrak{E}_{\infty}$ существует сильно голоморфная в секторе полугруппа, разрешаюшая уравнение $P(A) \dot{u}(t)=Q(A) u(t)$ и имеющая вид

$$
U(\tau) u_{0}=\int_{\sigma(A) \backslash \mathscr{P}_{0}} \exp \left(\frac{Q(\lambda)}{P(\lambda)} \tau\right) d E_{\lambda} u_{0}, \quad \tau \in \Sigma_{\theta}, \quad u_{0} \in \mathfrak{E}_{\infty}
$$

Ее ядро есть образ проектора $P=\int_{\mathscr{P}_{0}} d E_{\lambda}$, а фазовоепространство задачи Коши для уравнения - образ проектора $I-P$. Из теорем 8.1 и 9.1 сразу получаем

СлЕДСТВИЕ 9.1. Пусть в условиях теоремъ 9.1 вектор-функция $f: \overline{\mathbb{R}}_{+} \rightarrow \mathfrak{E}_{\infty}$ такова, что

$$
\begin{gathered}
\int_{\sigma(A) \cap \mathscr{P}_{0}} d E_{\lambda} f(t) \in C^{1}\left(\mathbb{R}_{+}, \mathfrak{E}_{\infty}\right) \cap C\left(\overline{\mathbb{R}}_{+}, \mathfrak{E}_{\infty}\right), \\
g(t)=\int_{\sigma(A) \backslash \mathscr{P}_{0}} d E_{\lambda} f(t) \text { удовлетворяет условиям леммьв 8.1, } \\
u_{0} \in\left\{u \in \mathfrak{E}_{\infty}: \int_{\sigma(A) \cap \mathscr{P}_{0}} Q(\lambda) d E_{\lambda} u=-\int_{\sigma(A) \cap \mathscr{P}_{0}} d E_{\lambda} f(0)\right\} .
\end{gathered}
$$


Тогда существует единственное решение и $\in C^{1}\left(\mathbb{R}_{+}, \mathfrak{E}_{\infty}\right) \cap C\left(\overline{\mathbb{R}}_{+}, \mathfrak{E}_{\infty}\right)$ ослабленной задачи Кочи

$$
u(0)=u_{0}, \quad P(A) \dot{u}(t)=Q(A) u(t)+f(t), \quad t \in \mathbb{R}_{+},
$$

имеющее вид

$$
\begin{aligned}
u(t)=- & \int_{\sigma(A) \cap \mathscr{P}_{0}} \frac{d E_{\lambda} f(t)}{Q(\lambda)}+\int_{\sigma(A) \backslash \mathscr{P}_{0}} \exp \left(\frac{Q(\lambda)}{P(\lambda)} t\right) d E_{\lambda} u_{0} \\
& +\int_{0}^{t} \int_{\sigma(A) \backslash \mathscr{P}_{0}} \exp \left(\frac{Q(\lambda)}{P(\lambda)}(t-s)\right) \frac{d E_{\lambda} f(s)}{P(\lambda)} d s .
\end{aligned}
$$

Полученньй результат используем при исследовании периодической задачи Коши для уравнения в частных производных со смешением по пространственной переменной. Для этого рассмотрим в пространстве $L_{2}(0,1)$ самосопряженный оператор

$$
A=i \frac{d}{d x}, \quad \operatorname{dom} A=\left\{v \in L_{2}(0,1): A v \in L_{2}(0,1), v(0)=v(1)\right\} .
$$

Его спектр состоит из собственных значений $\lambda_{k}=2 \pi k, k \in \mathbb{Z}$, которым соответствуют собственные функции $\varphi_{k}(x)=e^{-2 \pi k i x}$. Возьмем индуктивный предел $\mathfrak{E}_{\infty}$ шшкалы $\left\{\mathfrak{E}_{\tau}: \tau \in \mathbb{N}\right\}$, построенной по данному оператору $A \in \mathrm{Cl}\left(L_{2}(0,1)\right)$. Положим $L=P(A), M=R(A) e^{-i h A}$, где $P(\lambda), R(\lambda)$ - целые функции, $h \in \mathbb{R}$.

СлЕДСТВИЕ 9.2. Пусть $\mathfrak{U}=\mathfrak{F}=\mathfrak{E}_{\infty}, h \in \mathbb{R}$, целье функции $P(\lambda), R(\lambda)$ не имеют общих нулей в точках $2 \pi k, k \in \mathbb{Z}, u$, кроме того,

$$
\begin{gathered}
\exists a \in \mathbb{R}, \quad \exists \psi \in\left(0, \frac{\pi}{2}\right) \quad \forall k \in \mathbb{Z} \\
\frac{R}{P}(2 \pi k) e^{-2 \pi k i h} \subset\left\{\mu \in \mathbb{C}:|\arg (\mu-a)| \in\left[\frac{\pi}{2}+\psi, \pi\right]\right\} .
\end{gathered}
$$

Тогда оператор $R(A) e^{-i h A}$ сильно $(P(A), 0)$-секториален.

ДоказАтельство. Достаточно в теореме 9.1 взять оператор $A$ вида (9.4), $Q(\lambda)=R(\lambda) e^{-i h \lambda}$. Условие (9.1) выполняется в силу отсутствия у спектра $\sigma(A)$ конечных предельных точек.

ЗАмечАниЕ 9.2. Условие (9.5) выполняется, например, если $R(\lambda)$ - многочлен степени, не превосходящей степени многочлена $P(\lambda)$.

В условиях следствия 9.2 полугруппа операторов (9.3) имеет вид

$$
U(\tau) u_{0}=\sum_{P(2 \pi k) \neq 0} \exp \left(\frac{\tau e^{-2 \pi k i h} R(2 \pi k)}{P(2 \pi k)}\right) \int_{0}^{1} u_{0}(y) e^{2 \pi k i(y-x)} d y
$$

а ядром полугрупшы является подпространство $\mathfrak{U}^{0}=\overline{\operatorname{span}}\left\{e^{-2 \pi k i x}: P(2 \pi k)=0\right\}$. Заметим, что это подпространство может быть бесконечномерным.

В следующей формулировке использованы обозначения $P(\lambda)=\sum_{k=0}^{\infty} \widetilde{c}_{k} \lambda^{k}$, $R(\lambda)=\sum_{k=0}^{\infty} \widetilde{d}_{k} \lambda^{k}, c_{k}=i^{k} \widetilde{c}_{k}, d_{k}=i^{k} \widetilde{d}_{k}, k \in \mathbb{N}_{0},\langle u(y), v(y)\rangle=\int_{0}^{1} u(y) \overline{v(y)} d y$. 
ТЕОРема 9.2. Пусть в условиях следствия $9.2 f(x, t)=f(x+1, t)$ для всех $x \in \mathbb{R}, t \in \mathbb{R}_{+}$,

$$
\sum_{P(2 \pi k)=0}\left\langle f(y, t), e^{-2 \pi k i y}\right\rangle e^{-2 \pi k i x} \in C^{1}\left(\mathbb{R}_{+}, \mathfrak{E}_{\infty}\right) \cap C\left(\overline{\mathbb{R}}_{+}, \mathfrak{E}_{\infty}\right),
$$

вектор-функиия

$$
g(t)=\sum_{P(2 \pi k) \neq 0}\left\langle f(y, t), e^{-2 \pi k i y}\right\rangle e^{-2 \pi k i x}: \mathbb{R}_{+} \rightarrow \mathfrak{E}_{\infty}
$$

удовлетворяет условиям леммы 8.1,

$$
\begin{gathered}
u_{0} \in\left\{u \in \mathfrak{E}_{\infty}: R(2 \pi k) e^{-2 \pi k i h}\left\langle u(y), e^{-2 \pi k i y}\right\rangle=-\left\langle f(y, 0), e^{-2 \pi k i y}\right\rangle,\right. \\
P(2 \pi k)=0\} .
\end{gathered}
$$

Тогда для любъх $a, b \in \mathbb{R}, a<b$, существует единственное решение задачи

$$
\begin{aligned}
\sum_{l=0}^{\infty} c_{l} \frac{\partial^{l+1}}{\partial x^{l} \partial t} u(x, t) & =\sum_{l=0}^{\infty} d_{l} \frac{\partial^{l}}{\partial x^{l}} u(x+h, t)+f(x, t), \quad(x, t) \in(a, b) \times \mathbb{R}_{+}, \\
\frac{\partial^{l}}{\partial x^{l}} u(0, t) & =\frac{\partial^{l}}{\partial x^{l}} u(1, t), \quad l \in \mathbb{N}_{0}, \quad t \in \mathbb{R}_{+} \\
u(x, 0) & =\widetilde{u}_{0}(x),
\end{aligned}
$$

где $\widetilde{u}_{0}(x)$ - периодическое с периодом 1 продолжение на интервал $(a, b)$ функuии $u_{0}(x)$ с отрезка $[0,1]$. При этом решение имеет вид

$$
\begin{aligned}
u(x, t) & =-\sum_{P(2 \pi k)=0} \frac{e^{2 \pi k i h}}{R(2 \pi k)}\left\langle f(y, t), e^{-2 \pi k i y}\right\rangle e^{-2 \pi k i x}+U(t) u_{0} \\
& +\int_{0}^{t} \sum_{P(2 \pi k) \neq 0} \exp \left(\frac{(t-s) e^{-2 \pi k i h} R(2 \pi k)}{P(2 \pi k)}\right) \frac{\left\langle f(y, s), e^{-2 \pi k i y}\right\rangle e^{-2 \pi k i x}}{P(2 \pi k)} d s .
\end{aligned}
$$

ДоказАтельство. Решим задачу (9.6), (9.7) для уравнения

$$
P\left(i \frac{\partial}{\partial x}\right) \frac{\partial u}{\partial t}(x, t)=R\left(i \frac{\partial}{\partial x}\right) \exp \left(-i h i \frac{\partial}{\partial x}\right) u(x, t)+f(x, t)
$$

на множестве $[0,1] \times \mathbb{R}_{+}$. Она будет иметь решение указанного в теореме вида в силу следствия 9.2 и следствия 9.1 , в котором надо взять оператор $A$ вида (9.4) и $Q(\lambda)=R(\lambda) e^{-i h \lambda}$. Правая часть последнего уравнения имеет вид

$$
\sum_{l=0}^{\infty} d_{l} \frac{\partial^{l}}{\partial x^{l}} \sum_{s=0}^{\infty} \frac{h^{s}}{s !} \frac{\partial^{s}}{\partial x^{s}} u(x, t)+f(x, t)=\sum_{l=0}^{\infty} d_{l} \frac{\partial^{l}}{\partial x^{l}} u(x+h, t)+f(x, t) .
$$

Осталось полученное решение периодически продолжить по $x$ на $(a, b)$ в силу условия (9.6). 


\section{Список литературы}

1. Иосида К. Функциональнњй анализ. М.: Мир, 1967.

2. Хенри Д. Геометрическая теория полулинейных параболических уравнений. М.: Мир, 1985.

3. Клемент Ф., Хейманс Х., Ангенент С., ван Дуйн К., де Пахтер Б. Однопараметрические полугруппы. М.: Мир, 1992.

4. Костин B. A. К теореме Соломяка-Иосиды об аналитических полугруппах // Алгебра и анализ. 1999. Т. 11. № 1. С. 25-42.

5. Крейн С. Г. Линейные дифференциальные уравнения в банаховом пространстве. М.: Наука, 1967.

6. Демиденко Г. В., Успенский С. В. Уравнения и системы, не разрешенные относительно старшей производной. Новосибирск: Научная книга, 1998.

7. Favini A., Yagi A. Degenerate differential equations in Banach spaces. New York: Marcel Dekker Inc., 1999.

8. Егоров И.Е., Пятков С.Г., Попов С.В. Неклассические дифференциально-операторные уравнения. Новосибирск: Наука, 2000.

9. Свиридюк Г.А. К общей теории полугрупп операторов // УМН. 1994. Т. 49. № 4. C. $47-74$.

10. Свиридюк Г. А., Федоров В. Е. О единицах аналитических полугрупп операторов с ядрами // Сиб. матем. журн. 1998. Т. 39. №3. С. 604-616.

11. Гохберг И. Ц., Крейн М. Г. Введение в теорию линейных несамосопряженных операторов в гильбертовом пространстве. М.: Наука 1965.

12. Федоров B. E. Вьрожденные сильно непрерьвные полугрупшы операторов // Алгебра и анализ. 2000. Т. 12. №3. С. 173-200.

13. Зорич В. А. Математический анализ. II. М.: Наука, 1984.

14. Титчмари Е. Введение в теорию интегралов Фурье. М.: Гостехиздат, 1948.

15. Хилле Э., Филлипс Р. Функциональный анализ и полугруппы. М.: ИЛ, 1962.

16. Мизохата $C$. Теория уравнений с частными производными. М.: Мир, 1977.

17. Радыно Я. В. Линейные уравнения и борнология. Минск: Изд-во БГУ, 1982.

18. Волевич Л.Р., Гиндикин С.Г. Обобщенные функции и уравнения в свертках. М.: Физматлит, 1994.

Челябинский государственньй университет

Поступила в редакцию

E-mail : kar@csu.ru

25.07 .2003 и 30.01 .2004 